\title{
Two-level Factorial and Fractional Factorial Replicates in Blocks of Size Two
}

\author{
J.D. Godolphin \\ Department of Mathematics, University of Surrey, Guildford, Surrey. GU2 7XH, UK
}

\begin{abstract}
For $p$ two-level factors, designs comprising full replicates with runs in blocks of size two are investigated. The minimum number of replicates for estimation of all main effects and two-factor interactions is established and a construction method is developed based on replicate generators. Complete design classes are given in the minimum number of replicates for $p \leq 15$. Designs in full replicates are used as root designs to obtain designs in fractional $2^{p-r}$ replicates, again to estimate main effects and two-factor interactions, and designs are recommended for $p=4, \ldots, 15$. Guidance is given on design construction when only a subset of the interactions are of interest.

Keywords: Confounding; Design of Experiments; Factorial Effect; Fraction Generator; Replicate Generator.
\end{abstract}

\section{Introduction}

Two-level factorial and fractional factorial designs are widely used to identify significant effects in industrial processes. The occurrence of practical constraints, which can make it necessary to arrange runs in blocks of size two, provide a major motivation for work in this area. For example: it might only be possible to conduct two runs in a fixed time period; a batch of raw material or a machine may only accommodate two runs. Practical situations involving blocking in industrial experiments are described in Bisgaard (1994). A

Email address: j.godolphin@surrey.ac.uk (J.D. Godolphin) 
second motivation arises from two-color microarray experiments. Kerr (2006) gives a clear description of the use of designs with blocks of size two in such experiments.

Incorporating blocks of size two in a design is costly with respect to resources. For a $2^{p}$ experiment comprising replicates arranged in this way, Draper and Guttman (1997) establish that $p$ replicates are needed to estimate all factorial effects. Yang and Draper (2003) focus on estimation of main effects and two-factor interactions and construct designs for $p \leq 5$. Wang (2004) and Kerr (2006) seek designs to estimate all main effects and two-factor interactions for general $p$. Wang (2004) uses orthogonal arrays and gives constructions in $p-1$ replicates and $[(p+3) / 2]$ replicates, where $[\ldots]$ denotes the integer part of. Kerr (2006) gives a construction in $\left\lfloor\log _{2} p\right\rfloor+1$ replicates, where $\lfloor\ldots\rfloor$ denotes the floor of, and confirms by computer searches that designs cannot be obtained in fewer replicates for $p \leq 8$. Related work in Jacroux (2010) has a small number of full replicate designs.

There is relatively little work on designs in fractional replicates in blocks of size two. Key work includes Yang and Draper (2003), Wang (2004), Jacroux (2010) and Wang and Cook (2012). In these papers it is assumed that interactions involving three or more factors are negligible. The same assumptions are made for fractional designs in this work. Yang and Draper (2003) and Wang (2004) give designs in half replicates. Jacroux (2010) provides constructions in $p$ and $p-1$ fractional replicates. Wang and Cook (2012) estimate effects of interest by combining information from half factorial replicates for $4 \leq p \leq 8$.

Cheng et al. (2004) and Jacroux (2009) consider blocking in non-regular fractional replicates. Such designs offer more flexibility over the number of runs, but have a complex aliasing structure. In this work, attention is restricted to regular fractional factorials: within a fractional factorial, any pair of effects will either be orthogonal or totally aliased.

A Design Class Construction Process (DCCP) is developed to obtain all designs in $M$ blocked $2^{p}$ replicates, with blocks of size two, which enable estimation of all main effects and two-factor interactions. A commonality between the process and the Kerr (2006) construction is the use of replicate generators. The novelty of this work is in the 
representation of designs in $M$ replicates by $p \times M$ replicate generator matrices, and the association of these matrices with columns of an $M \times\left(2^{M}-1\right)$ matrix. The DCCP operates by seeking replicate generator matrices with specific properties regarding the columns. If these properties are achieved then the $M$ rows of the matrix correspond to $M$ replicates such that each main effect and each two factor interaction is estimable from at least one replicate. The approach has several advantages: for given $p$ and $M$ the number of potential designs is much smaller than that checked by the computer search of Kerr (2006); the method of design generation is systematic and incorporates recognition of isomorphic designs to avoid double counting, features not shared by other methods. These properties enable complete sets of designs in $M$ replicates to be identified for given $p$. As a further benefit of the approach, the structure of the underlying $M \times\left(2^{M}-1\right)$ matrix gives insight into the range of design properties, for given $p, M$ pair.

Unless $p$ is small, the number of runs required for several replicates is likely to exceed the resources available. The need to limit the number of runs is taken into account of in two ways, which can be combined. First, restricting estimation of the interactions to a subset of the two-factor interactions may allow the use of fewer replicates. Second, designs in $M$ blocked $2^{p-r}$ replicates from which all effects of interest are estimable can be constructed. Both approaches can keep the number of runs to a manageable number.

Fundamental concepts are introduced in $\S 2$. The DCCP is developed in $\S 3$ and it is established that the number of replicates used in the Kerr (2006) construction is the minimum to achieve estimation of all main effects and two factor interactions. Complete design classes in the minimum number of replicates are found for $p \leq 15$ in $\S 4$ using Matlab R2017a. Complete design classes do not appear to have been produced previously for $p>4$. Guidance is given on construction of designs in fewer replicates to estimate all main effects but only selected interactions in $\S 5$. In $\S 6$ and $\S 7$ designs in blocked $2^{p}$ replicates are used to form designs in blocked $2^{p-r}$ replicates and a systematic approach exploits the availability of complete design classes to yield up to four fractional designs for given $p$, with 
designs involving successively larger $r$, i.e. smaller fractions, and larger $M$. Designs are recommended for $4 \leq p \leq 15$ and constructions are given for two series of designs. In many cases, the recommended designs have fewer runs and favourable estimibility properties compared to designs already available in the literature. Finally, guidance is given on construction of designs in fractional replicates to estimate all main effects and selected interactions.

\section{Preliminaries}

Notation is consistent with Chapters 6 to 8 of Montgomery (2012). The factors in a $2^{p}$ experiment are $A, B, \ldots$, and this notation is also used for the $p$ main effects. The $p(p-1) / 2$ two-factor interactions are denoted $A B, A C, \ldots, B C, \ldots$ For brevity, unless otherwise stated, interaction will be taken to mean a two-factor interaction. Treatment combinations are expressed in lower case letters. For example, ad represents a run with $A$ and $D$ high and all other factors low. The treatment combination with every factor low is denoted by (1). A replicate involves a run in each of the $2^{p}$ treatment combinations. A factorial effect partitions the treatment combinations into two sets of size $2^{p-1}$. Combinations in the first set have an odd number of effect factors at low level and those in the second set have an even number at low level. The factorial effect is estimated by the subtraction of the average of the first set from that of the second.Thus, an estimator of a factorial effect, based on one replicate, is a linear combination of the $2^{p}$ observations, with coefficients $\pm 1 / 2^{p-1}$. The estimator has variance $\sigma^{2} / 2^{p-2}$, where $\sigma^{2}$ is the common variance of the observations.

The $2^{p}$ treatment combinations can be applied to the experimental units in $2^{p-1}$ blocks of size two so that $2^{p-1}$ factorial effects are orthogonal to blocks, and are estimable, and the remaining effects are completely confounded with blocks, and are inestimable. Such an arrangement will be described as a blocked replicate. It is assumed that blocks do not interact with factors. The model is

$$
y_{i j k}=\mu+\tau_{i}+\beta_{j k}+\rho_{k}+\epsilon_{i j k}
$$


Here, $y_{i j k}$ is the observation from application of the $i$ th treatment combination to an experimental unit in the $j$ th block of the $k$ th replicate. The overall mean is $\mu$ and $\tau_{i}$, $\beta_{j k}$ and $\rho_{k}$ are the effects of the $i$ th treatment combination, the $j$ th block nested in the $k$ th replicate and the $k$ th replicate. The error terms $\epsilon_{i j k}$ are uncorrelated, all with variance $\sigma^{2}$.

\subsection{Example of a Blocked $2^{4}$ Replicate}

A blocked $2^{4}$ replicate with columns appertaining to the eight blocks is given by:

\begin{tabular}{|c|c|c|c|c|c|c|c|}
\hline 1 & 2 & 3 & 4 & 5 & 6 & 7 & 8 \\
\hline$(1)$ & $a$ & $b$ & $a b$ & $d$ & $a d$ & $b d$ & $c d$ \\
$a b c$ & $b c$ & $a c$ & $c$ & $a b c d$ & $b c d$ & $a c d$ & $a b d$ \\
\hline
\end{tabular}

Blocking comes at considerable cost to the estimation capacity of the replicate. Without blocking, all fifteen effects would be estimable from the sixteen runs. Due to the specific pairing of treatment combinations in blocks, $A B, A C, B C, D, A B D, A C D$ and $B C D$ are confounded with blocks and are inestimable. For a confounded effect, each block has both treatment combinations with an even number of effect factors at low level, or both with an odd number of effect factors at low level. For example, consider $A B$ : treatment combinations in blocks 1, 4, 5 and 8 all have an even number of $A, B$ at low level whereas those in blocks 2, 3, 6 and 7 all have an odd number of $A, B$ at low level. The remaining factorial effects: $A, B, C, A B C, A D, B D, C D, A B C D$, are othogonal to blocks and are estimable. For each of these, every block contains one treatment combination with an odd number of effect factors at low level and one with an even number of effect factors at low level.

\section{2. replicate generators}

The block containing (1) is the principal block. Once the treatment combination to be paired with (1) in the principal block has been chosen, the remaining blocks are constructed to contain cosets of the combinations in this block. Kerr (2006) uses the term generator for the treatment combination paired with (1). In this work the term replicate generator is 
used to avoid confusion with block generators and with generators for fractional replicates. The estimability properties of the blocked replicate are obtained directly from the replicate generator: the $2^{p-1}$ estimable effects are those effects with an odd number of constituent factors at high level in the replicate generator. Referring back to the example of $\S 2.1$, the eight blocks of the replicate can be constructed from the replicate generator $a b c$, and the estimable factorial effects are those containing an odd number of $A, B, C$.

It might be anticipated that, with careful choice of replicate generator, one blocked $2^{p}$ replicate would be sufficient to estimate all $p$ main effects and $p(p-1) / 2$ interactions. This is not the case. The need for more than one replicate is demonstrated by reference again to a $2^{4}$ factorial. To estimate all main effects from a single blocked $2^{4}$ replicate, the replicate generator must be abcd. However, this would not enable estimation of any interactions, since each of these has an even number of terms in common with abcd.

The $q$ factors at high level in the replicate generator of a blocked replicate are described as selected, and the remaining $p-q$ factors as non-selected. Main effects of the selected factors are estimable from the replicate, as are the $q(p-q)$ interactions involving one selected and one non-selected factor. Selected factors are said to be contained in the replicate generator, and the replicate generator is said to have length $q$. Designs can be constructed from a number of blocked replicates. Estimators of main effects and interactions from each replicate are pooled. An effect which is estimable from $n$ blocked replicates has an estimator which is the average of the $n$ individual estimators and has variance $\sigma^{2} /\left(n 2^{p-2}\right)$.

The class of designs in $M$ blocked $2^{p}$ replicates, from which all main effects and interactions are estimable is denoted $\mathcal{D}_{M}^{p}$. The aim is to identify all members of $\mathcal{D}_{M}^{p}$.

\section{Design Construction}

\subsection{Generator Matrices and Conditions}

Consider design $\mathcal{D}$, not necessarily in $\mathcal{D}_{M}^{p}$, comprising $M$ blocked $2^{p}$ replicates. The generator of the $i$ th replicate, $\mathrm{g}_{i}$, has length $q_{i}$ and can be represented by $\left(x_{i 1}, x_{i 2}, \cdots, x_{i p}\right)$, where $x_{i j}=1$ if $g_{i}$ contains the $j$ th factor and $x_{i j}=0$ otherwise. It follows that $\Sigma_{j=1}^{p} x_{i j}=$ 
$q_{i}>0$. For example, the replicate generator $a b c$ for the blocked $2^{4}$ replicate of $\S 2.1$ has representation $(1,1,1,0)$. Design $\mathcal{D}$ is specified by the $M \times p$ generator matrix $X^{\mathcal{D}}$ :

$$
X^{\mathcal{D}}=\left(\begin{array}{cccc}
x_{11} & x_{12} & \cdots & x_{1 p} \\
x_{21} & x_{22} & \cdots & x_{2 p} \\
\vdots & \vdots & & \vdots \\
x_{M 1} & x_{M 2} & \cdots & x_{M p}
\end{array}\right) .
$$

The generator matrix depiction of (3.1) will be used to obtain all designs in $\mathcal{D}_{M}^{p}$. Some matrix notation is introduced towards this aim. Let the $M \times\left(\begin{array}{l}M \\ m\end{array}\right)$ matrix $X_{M m}$ contain all columns comprising $m$ elements zero and $M-m$ elements unity, for $m=0, \ldots, M-1$, and define the $M \times\left(2^{M}-1\right)$ matrix $X_{M}=\left(X_{M 0} \ldots X_{M M-1}\right)$. Matrices $X_{1}$ to $X_{4}$ are:

$$
\begin{gathered}
X_{1}=(1), \quad X_{2}=\left(\begin{array}{lll}
1 & 1 & 0 \\
1 & 0 & 1
\end{array}\right), \quad X_{3}=\left(\begin{array}{llllllll}
1 & 1 & 1 & 0 & 1 & 0 & 0 \\
1 & 1 & 0 & 1 & 0 & 1 & 0 \\
1 & 0 & 1 & 1 & 0 & 0 & 1
\end{array}\right) \\
X_{4}=\left(\begin{array}{lllllllllllllll}
1 & 1 & 1 & 1 & 0 & 1 & 1 & 1 & 0 & 0 & 0 & 1 & 0 & 0 & 0 \\
1 & 1 & 1 & 0 & 1 & 1 & 0 & 0 & 1 & 1 & 0 & 0 & 1 & 0 & 0 \\
1 & 1 & 0 & 1 & 1 & 0 & 1 & 0 & 1 & 0 & 1 & 0 & 0 & 1 & 0 \\
1 & 0 & 1 & 1 & 1 & 0 & 0 & 1 & 0 & 1 & 1 & 0 & 0 & 0 & 1
\end{array}\right)
\end{gathered}
$$

Theorem 1. $\mathcal{D} \in \mathcal{D}_{M}^{p}$ iff the following conditions are both satisfied:

(i) The $p$ columns of $X^{\mathcal{D}}$ are all different columns of $X_{M}$;

(ii) Each row of $X^{\mathcal{D}}$ contains at least one non-zero term.

Proof: A main effect is estimable iff the factor is contained in at least one replicate generator. This occurs iff the corresponding column of $X^{\mathcal{D}}$ contains at least one element unity, i.e., is a column of $X_{M}$. An interaction is estimable iff at least one replicate generator contains exactly one of the two factors, i.e. the corresponding columns of $X^{\mathcal{D}}$ are different. Thus condition (i) holds iff all effects of interest are estimable. The rows of $X^{\mathcal{D}}$ represent 
$M$ valid blocked replicates iff each replicate generator is a treatment combination other than (1) which occurs iff $X^{\mathcal{D}}$ contains at least one non-zero term. The result follows.

If $\mathcal{D} \in \mathcal{D}_{M}^{p}$ then, by Theorem $1, X^{\mathcal{D}}$ comprises $p$ distinct columns of $X_{M}$. Conversely, any $M \times p$ matrix of $p$ distinct columns of $X_{M}$, such that each row sum is non-zero, corresponds to a design in $\mathcal{D}_{M}^{p}$. For $X_{M}$ to contain at least $p$ columns, it is necessary that $2^{M}-1 \geq p$, that is, $M \geq M_{0}=\left\lceil\log _{2}(p+1)\right\rceil$, where $\lceil\ldots\rceil$ denotes the ceiling of. Since $2^{M_{0}-1} \leq p \leq 2^{M_{0}}-1$, every row of $X_{M_{0}}$ contains $2^{M_{0}-1}-1<p$ zero terms. Thus, any matrix comprising $p$ columns of $X_{M_{0}}$ has at least one non-zero term and, by Theorem 1 , is a generator matrix for a design in $\mathcal{D}_{M_{0}}^{p}$. Thus, a corollary to Theorem 1 is:

Corollary 1. At least $M_{0}=\left\lceil\log _{2}(p+1)\right\rceil$ blocked replicates are required for estimation of all main effects and interactions. $\mathcal{D} \in \mathcal{D}_{M_{0}}^{p}$ iff $X^{\mathcal{D}}$ comprises $p$ distinct columns from $X_{M_{0}}$.

The lower bound of $M_{0}$ blocked $2^{p}$ replicates for a design from which all effects of interest are estimable is equivalent to the bound noted in $\operatorname{Kerr}(2006)$, for $p \leq 8$. For $M \geq M_{0}$, the use of $X_{M}$ to obtain designs in $\mathcal{D}_{M}^{p}$ is now demonstrated.

\subsection{Examples of Designs in Blocked $2^{4}$ Replicates}

From Corollary 1, for $p=4$ at least $M_{0}=\left\lceil\log _{2} 5\right\rceil=3$ blocked replicates are required to estimate all effects of interest. Any four columns from $X_{3}$ give a generator matrix for a design in $\mathcal{D}_{3}^{4}$. Let $\mathcal{D} 1$ have generator matrix comprising columns $1,2,3,5$ and $\mathcal{D} 2$ have generator matrix formed from columns 2,5,6,7:

$$
X^{\mathcal{D} 1}=\left(\begin{array}{cccc}
1 & 1 & 1 & 1 \\
1 & 1 & 0 & 0 \\
1 & 0 & 1 & 0
\end{array}\right) \text { and } X^{\mathcal{D} 2}=\left(\begin{array}{cccc}
1 & 1 & 0 & 0 \\
1 & 0 & 1 & 0 \\
0 & 0 & 0 & 1
\end{array}\right) \text {. }
$$

The sets of replicate generators for $\mathcal{D} 1$ and $\mathcal{D} 2$ are read directly from the rows of $X^{\mathcal{D} 1}$ and $X^{\mathcal{D} 2}$ as $\{a b c d, a b, a c\}$ and $\{a b, a c, d\}$. The 24 blocks of each design can be constructed from these sets. If resources are available for more than three replicates, the appropriate $X_{M}$ matrix is used. To construct designs in four blocked replicates, say, any four columns 
of $X_{4}$ such that each row sum is non-zero, give a generator matrix for a design in $\mathcal{D}_{4}^{4}$. For example, columns 5,6,7,8 give the design with replicate generator set $\{b c d, a b, a c, a d\}$.

\subsection{Isomorphism Types}

There are two ways in which $p$ columns from $X_{M}$ give isomorphic designs. These are categorised as follows:

Type I Isomorphism: Designs $\mathcal{D} \mathcal{A}$ and $\mathcal{D B}$ in $\mathcal{D}_{M}^{p}$ are said to be isomorphic of Type I if $X^{\mathcal{D A}}$ and $X^{\mathcal{D B}}$ comprise the same $p$ columns of $X_{M}$ ordered differently.

Type II Isomorphism: Designs $\mathcal{D} \mathcal{A}$ and $\mathcal{D} \mathcal{B}$ in $\mathcal{D}_{M}^{p}$ are said to be isomorphic of Type II if they are not isomorphic of Type I and the factor labels in the generator set for $\mathcal{D} \mathcal{A}$ can be rearranged to give the generator set for $\mathcal{D} \mathcal{B}$.

The isomorphism types are demonstrated by reference to designs in $\mathcal{D}_{3}^{4}$ considered in $\S 3.2$. For an example of a Type I isomorphism, let design $\mathcal{D} 3$ have $X^{\mathcal{D} 3}$ comprising columns $5,2,3,1$ of $X_{3}$, in that order. These are the columns used for $X^{\mathcal{D} 1}$ of (3.2), with first and last columns interchanged. Thus, $\mathcal{D} 1$ and $\mathcal{D} 3$ are Type I isomorphic. The replicate generator set for $\mathcal{D} 3$ is $\{a b c d, b d, c d\}$, which becomes the generator set for $\mathcal{D} 1$ on interchanging $a$ and d. To illustrate Type II isomorphism, let $\mathcal{D} 4$ have generator matrix formed from columns $3,5,6,7$ of $X_{3}$, in that order. These are different to the columns used in $X^{\mathcal{D} 2}$ of $(3.2)$, but switching the last two rows and the last two columns of $X^{\mathcal{D} 4}$ gives $X^{\mathcal{D} 2}$. Thus, $\mathcal{D} 4$, with replicate generator set $\{a b, c, a d\}$, is Type II isomorphic to $\mathcal{D} 2$, with the permutation in factor labels in the generator set for $\mathcal{D} 4$ required to give the generator set for $\mathcal{D} 2$ being the interchange of $c$ and $d$.

\subsection{Identification of all Designs in $\mathcal{D}_{M}^{p}$ : the DCCP}

By considering each set of $p$ columns from $X_{M}$ as a potential generator matrix, where $M \geq M_{0}$, every design in $\mathcal{D}_{M}^{p}$ will be identified. However, many designs will be isomorphic and a strategy is required to avoid multiple listing of designs.

To find a full set of non-isomorphic designs in $\mathcal{D}_{M}^{p}$ for $p, M$ pair, with $M \geq M_{0}$, the DCCP systematically inspects every set of $p$ columns from $X_{M}$ as a potential generator 
matrix. By Corollary 1 , for $M=M_{0}$ every such matrix generates a design in $\mathcal{D}_{M_{0}}^{p}$. By Theorem 1 , for $M>M_{0}$ any matrix with every row sum exceeding zero generates a design in $\mathcal{D}_{M}^{p}$. Type I isomorphisms do not occur since a set of $p$ columns is only considered once. For consistency, columns are ordered in the generator matrix in the order in which they occur in $X_{M}$.

The avoidance of Type II isomorphisms is more challenging. A design, $\mathcal{D}$ say, in $\mathcal{D}_{M}^{p}$ is only recorded if no permutation of rows and columns maps $X^{\mathcal{D}}$ onto the generator matrix of a design already recorded. In practice, it is only necessary to consider mapping $X^{\mathcal{D}}$ onto generator matrices of designs already listed which have the same distribution of columns amongst $X_{M 0}, \ldots, X_{M M-1}$. For example, consider $\mathcal{D} 5, \mathcal{D} 6$ and $\mathcal{D} 7$ of $\mathcal{D}_{4}^{8}$ with generator matrices:

$$
\begin{aligned}
X^{\mathcal{D} 5} & =\left(\begin{array}{llllllll}
1 & 1 & 1 & 0 & 1 & 0 & 0 & 0 \\
1 & 1 & 0 & 1 & 0 & 1 & 0 & 0 \\
1 & 0 & 1 & 0 & 0 & 0 & 1 & 0 \\
0 & 1 & 0 & 1 & 0 & 0 & 0 & 1
\end{array}\right) \\
X^{\mathcal{D} 6} & =\left(\begin{array}{llllllll}
1 & 0 & 1 & 1 & 1 & 0 & 0 & 0 \\
0 & 1 & 0 & 0 & 0 & 1 & 0 & 0 \\
1 & 1 & 1 & 0 & 0 & 0 & 1 & 0 \\
1 & 1 & 0 & 1 & 0 & 0 & 0 & 1
\end{array}\right) \\
X^{\mathcal{D} 7} & =\left(\begin{array}{llllllll}
1 & 0 & 1 & 0 & 0 & 0 & 0 & 0 \\
1 & 1 & 0 & 1 & 1 & 1 & 0 & 0 \\
1 & 1 & 0 & 1 & 0 & 0 & 1 & 0 \\
1 & 1 & 1 & 0 & 1 & 0 & 0 & 1
\end{array}\right) .
\end{aligned}
$$

Matrices $X^{\mathcal{D} 5}$ and $X^{\mathcal{D} 6}$ have common profile with each containing two columns from $X_{41}$, two columns from $X_{42}$, and all columns of $X_{43}$. Matrix $X^{\mathcal{D} 7}$ has a different profile since it comprises the column from $X_{40}$, one column from $X_{41}$ and three columns from each of $X_{42}$ 
and $X_{43}$. Thus $\mathcal{D} 7$ cannot be isomorphic to $\mathcal{D} 5$ or $\mathcal{D} 6$. In general, for two designs with common distributions of generator matrix columns, such as $\mathcal{D} 5$ and $\mathcal{D} 6$, to determine if there is a permutation mapping one generator matrix onto the other, column permutations can be restricted to within partial $X_{M i}$. Thus, far fewer than $p ! \times M !$ permutations are required. To determine if there is a permutation mapping $X^{\mathcal{D} 5}$ onto $X^{\mathcal{D} 6}$ it is sufficient to consider just the first four columns of each generator matrix. None of the $2 \times 2 \times 24$ permutations between the first two columns, the third and fourth columns and the rows of the sub-matrix of $X^{\mathcal{D} 5}$ maps onto the sub-matrix of $X^{\mathcal{D} 6}$ and so the designs are not Type II isomorphic.

Being able to efficiently recognise designs which are Type II isomorphic, as described above, is fundamental to the DCCP because even for $M$ as small as 4 , the number of designs can become very large, as will be seen in $§ 4$.

\subsection{Design Ranking}

There can be considerable diversity in estimability properties within a $\mathcal{D}_{M}^{p}$. For example, for the 365 designs in $\mathcal{D}_{4}^{8}$, the average variance of main effect estimators takes values from $0.1875 \sigma^{2}$ to $0.44 \sigma^{2}$. A ranking for designs in $\mathcal{D}_{M}^{p}$ is proposed to aid with design selection. This is particularly useful for $\mathcal{D}_{M}^{p}$ with large cardinality, such as $\mathcal{D}_{4}^{8}$. The ranking prioritises estimation of main effects over interactions: a strategy consistent with comments made in Yang and Draper (2003) and Kerr (2006).

For $\mathcal{D} \in \mathcal{D}_{M}^{p}$, let $N(\mathcal{D})$ be the total number of main effect estimates from the $M$ replicates and $n_{i}(\mathcal{D})$ be the number of main effects with estimates from $i$ replicates. Similarly, define $T(\mathcal{D})$ and $t_{i}(\mathcal{D})$ for estimates of interactions. Thus $N(\mathcal{D})=\Sigma_{i=1}^{M} i n_{i}(\mathcal{D})$ and $T(\mathcal{D})=\sum_{i=1}^{M} i t_{i}(\mathcal{D})$. For example, $\mathcal{D} 1$ of $\S 3.2$, with replicate generator set $\{a b c d, a b, a c\}$, yields estimates of $A$ from all replicates, $B$ and $C$ from two replicates each, and $D$ from one replicate. Thus, $N(\mathcal{D} 1)=8, n_{1}(\mathcal{D} 1)=1, n_{2}(\mathcal{D} 1)=2$ and $n_{3}(\mathcal{D} 1)=1$. Estimates of $A B, A C, B D$ and $B C$ are each given from one replicate, whilst estimates of $A D$ and $B C$ each arise from two replicates, giving $T(\mathcal{D} 1)=8, t_{1}(\mathcal{D} 1)=4$ and $t_{2}(\mathcal{D} 1)=2$. The 
proposed ranking is now stated.

For $\mathcal{D} \mathcal{A}$ and $\mathcal{D B}$ in $\mathcal{D}_{M}^{p}$, design $\mathcal{D} \mathcal{A}$ has higher ranking if one of the following is true:

(i) $N(\mathcal{D} \mathcal{A})>N(\mathcal{D B})$

(ii) $N(\mathcal{D} \mathcal{A})=N(\mathcal{D B}), n_{i}(\mathcal{D} \mathcal{A})=n_{i}(\mathcal{D B})$ for $i=1, \ldots, j-1$, and $n_{j}(\mathcal{D} \mathcal{A})<n_{j}(\mathcal{D B})$

(iii) $n_{i}(\mathcal{D A})=n_{i}(\mathcal{D B})$ for $i=1, \ldots, M$ and $T(\mathcal{D} \mathcal{A})>T(\mathcal{D B})$

(iv) $n_{i}(\mathcal{D} \mathcal{A})=n_{i}(\mathcal{D B})$ for $i=1, \ldots, M, T(\mathcal{D} \mathcal{A})=T(\mathcal{D B}), t_{i}(\mathcal{D A})=t_{i}(\mathcal{D B})$ for $i=$ $1, \ldots, j-1$, and $t_{j}(\mathcal{D A})<t_{j}(\mathcal{D B})$

Part (i) of the scheme ranks designs with a larger number of main effect estimates above those with a smaller number. For designs with the same number of main effect estimates, part (ii) favours designs where fewer main effects have estimates from only a small number of replicates, that is, fewer estimates of main effects have poor efficiency. For designs with common attributes for main effect estimates, parts (iii) and (iv) compare the quality of interaction estimates.

\section{Designs in $M_{0}$ Blocked Replicates}

The DCCP of $\S 3$ is used to generate $\mathcal{D}_{M_{0}}^{p}$ for $p=2, \ldots, 15$ and to rank the designs in each class. The design classes and rankings are otained with Matlab R2017a.

4.1. $\mathcal{D}_{M_{0}}^{p}$ classes for $p \leq 15$

$\boldsymbol{M}_{\mathbf{0}}=\mathbf{2}$ : Estimation of all effects of interest requires $M_{0}=2$ blocked replicates for $p=2,3$. Design class $\mathcal{D}_{2}^{2}$ contains two designs. The generator sets and design properties are reported in Table 1. The design with replicate generator set $\{a b, a\}$ has $N=3$ with estimates of $A$ from both replicates and $B$ from one replicate. This is ranked above the design with generator set $\{a, b\}$ which, with one estimate of each main effect, has $N=2$. There is only one design in $\mathcal{D}_{2}^{3}$ and this uses all three columns of $X_{2}$. See Table 2 for details. Yang and 
Table 1: $p=2$, designs in $\mathcal{D}_{2}^{2}$

\begin{tabular}{ccccccccccc}
\hline $\mathrm{rg}^{a}$ sets & $A$ & $B$ & $A B$ & $N$ & $n_{1}$ & $n_{2}$ & $T$ & $t_{1}$ & $t_{2}$ & ranking \\
\hline$a b, a$ & 2 & 1 & 1 & 3 & 1 & 1 & 1 & 1 & 0 & 1 \\
$a, b$ & 1 & 1 & 2 & 2 & 2 & 0 & 2 & 0 & 1 & 2 \\
\hline
\end{tabular}

${ }^{a}$ replicate generator

Table 2: $p=3$, design in $\mathcal{D}_{2}^{3}$

\begin{tabular}{cccccccccccccc}
\hline $\mathrm{rg}^{a}$ sets & $A$ & $B$ & $C$ & $A B$ & $A C$ & $B C$ & $N$ & $n_{1}$ & $n_{2}$ & $T$ & $t_{1}$ & $t_{2}$ & ranking \\
\hline$a b, a c$ & 2 & 1 & 1 & 1 & 1 & 2 & 4 & 2 & 1 & 4 & 2 & 1 & 1 \\
\hline
\end{tabular}

${ }^{a}$ replicate generator

Draper (2003) give the designs of $\mathcal{D}_{2}^{2}$ and $\mathcal{D}_{2}^{3}$ in a different format. The design in $\mathcal{D}_{2}^{3}$ is also identified by Kerr (2006) and by Method 2 of Jacroux (2010).

$\boldsymbol{M}_{\mathbf{0}}=3$ : For $p=4, \ldots, 7$, the minimum number of blocked replicates for estimation of all effects of interest is $M_{0}=3$. Designs in $\mathcal{D}_{3}^{p}$ have generator matrices comprising $p$ columns from $X_{3}$. Due to space considerations, only the replicate generator sets, $N, n_{i}, T, t_{i}$ and rankings are reported. There are ten designs in $\mathcal{D}_{3}^{4}$ : see Table A.10. Designs $\mathcal{D} 1$ and $\mathcal{D} 2$ of $\S 3.2$ are ranked third and tenth respectively. Yang and Draper (2003) give the ten designs in an alternative format. Kerr (2006) finds all the designs of $\mathcal{D}_{3}^{4}$, but duplicates two designs. For $p=4$, the constructions of Wang (2004) give designs ranked first and third. Method 2 of Jacroux (2010) gives designs ranked seventh and ninth. Classes $\mathcal{D}_{3}^{5}, \mathcal{D}_{3}^{6}$ and $\mathcal{D}_{3}^{7}$ have cardinalities six, three and one respectively. See Table A.11. For $p>4$, the constructions in Wang (2004) require more than $M_{0}$ blocked replicates, and Method 2 of Jacroux (2010) gives fractional designs. Neither Yang and Draper (2003) nor Kerr (2006) give information on design classes for $p>4$.

$M_{0}=4:$ For $p=8, \ldots, 15$, the value of $M_{0}$ is four. Generator matrices comprise $p$ columns of $X_{4}$. The cardinality of each $\mathcal{D}_{4}^{p}$ is given in Table 3. Tables A.12 to A.16 give the 15

Table 3: Number of Designs in $\mathcal{D}_{4}^{p}$

\begin{tabular}{ccccccccc}
\hline$p$ & 8 & 9 & 10 & 11 & 12 & 13 & 14 & 15 \\
No. of designs & 365 & 290 & 187 & 97 & 39 & 13 & 4 & 1 \\
\hline
\end{tabular}


highest ranked designs for $p=8, \ldots, 12$. In each case, the lowest ranked design is also presented, to indicate the breadth of properties in the class. Table A.17 contains all designs for $p=13,14$ and 15. The design ranked $33 \mathrm{rd}$ in $\mathcal{D}_{4}^{8}$ is given by the general construction of Kerr (2006) and is included in Table A.12 for comparison. The slightly weaker estimation of main effects for this design in comparison to the higher ranked designs, is seen by inspection of $N$ and $n_{1}$ values. With $T=48$, the design has lowest $T$ value of all designs in $\mathcal{D}_{4}^{8}$.

For $p=8, \ldots, 12$ a list of all designs in $\mathcal{D}_{4}^{p}$ is available on application to the author.

\subsection{General Observations on $\mathcal{D}_{M_{0}}^{p}$ Classes}

For given $M_{0}$, the number of factors takes values from $2^{M_{0}-1}$ to $2^{M_{0}}-1$. It is informative to examine trends in properties of $\mathcal{D}_{M_{0}}^{p}$ as $p$ moves through this range. There are $\left(\begin{array}{c}2^{M_{0}}-1 \\ p\end{array}\right)$ selections of columns from $X_{M_{0}}$ to form generator matrices for designs in $\mathcal{D}_{M_{0}}^{p}$, before isomorphic designs are discounted. This number decreases as $p$ increases, and the same trend is observed in the $\mathcal{D}_{4}^{p}$ cardinalities in Table 3. From the DCCP, it is evident that there is a single design for $p=2^{M_{0}}-1$ and this has all columns of $X_{M_{0}}$ in the generator matrix. For $p=2^{M_{0}}-2$ there are $M_{0}$ designs, each using all but one column of $X_{M_{0}}$ in the generator matrix: the $M_{0}$ designs relate to the $M_{0}$ submatrices that the omitted column can be selected from. These features are noted for $M_{0}=2,3,4$, in tables of designs.

Consider $\mathcal{D} \in \mathcal{D}_{M_{0}}^{p}$. Then $N(\mathcal{D})$ can be expressed in terms of the generator matrix as:

$$
N(\mathcal{D})=1_{M_{0}}^{T} X^{\mathcal{D}} 1_{M_{0}}
$$

where $1_{M_{0}}$ denotes the $M_{0} \times 1$ vector with all terms unity. From $(4.1)$, high values of $N(\mathcal{D})$ are obtained when the columns for $X^{\mathcal{D}}$ are predominantly from the left hand side of $X_{M_{0}}$ and low values when the columns are predominantly from the right hand side. The two designs with $X^{\mathcal{D}}$ comprising the first and last $p$ columns of $X_{M_{0}}$ have the highest and lowest $N(\mathcal{D})$. For example, for $p=8$ the first eight and last eight columns of $X_{4}$ have one common column and the sums of terms in the two sets of columns are 22 and 12 respectively, giving the highest and lowest values of $N$ in $\mathcal{D}_{4}^{8}$. As $p$ increases, so does the overlap between the 
first and last $p$ columns of $X_{M_{0}}$ and the range in $N$ decreases within a class. Thus, for given $M_{0}$, the $\mathcal{D}_{M_{0}}^{p}$ classes with larger cardinalities are those exhibiting the greatest range in $N$. For such $\mathcal{D}_{M_{0}}^{p}$, the design ranking is particularly useful to aid in design selection.

A comparison can be made between design properties favouring estimation of main effects and those favouring estimation of interactions. Since each row of $X_{M_{0}}$ has $2^{M_{0}-1}-1$ zero terms and $2^{M_{0}-1}$ unity terms, every replicate generator satisfies $p-2^{M_{0}-1}+1 \leq q_{i} \leq$ $2^{M_{0}-1}$. In terms of the replicate generator lengths, $N(\mathcal{D})$ and $T(\mathcal{D})$ are:

$$
N(\mathcal{D})=\sum_{i=1}^{M_{0}} q_{i} \text { and } T(\mathcal{D})=\sum_{i=1}^{M_{0}} q_{i}\left(p-q_{i}\right) .
$$

From $(4.2)$, high $q_{i}$ favour high $N(\mathcal{D})$ and $q_{i}$ close to $p / 2$ favour high $T(\mathcal{D})$. Thus, maximising $N(\mathcal{D})$ is not compatible with maximising $T(\mathcal{D})$, except for $p=2^{M_{0}}-1$ where

there is one design and all $q_{i}$ are $p / 2=2^{M_{0}-1}$. For example, nine designs in $\mathcal{D}_{4}^{8}$ have all $q_{i}=p / 2=4$, giving a maximum value of 64 for $T$. With $N=16$, these designs provide considerably fewer main effect estimates than the top ranked designs in Table A.12. In fact the designs with $T=64$ are not even ranked amongst the top 150 designs in the class.

Some comment should be made on the general construction method of Kerr (2006). As noted, the cardinalities of $\mathcal{D}_{M_{0}}^{p}$ classes for $p=2^{s}$ seem to be particularly large. For such $p$, the method of Kerr generates just one design from the class. For $p$ not a power of two, the general construction enables easy construction of designs in $\mathcal{D}_{M_{0}}^{p}$. However, the method does not give a systematic approach to enable construction of all designs in a class and provides no information on the number of designs available or guidance on selection.

\section{Designs in Fewer than $M_{0}$ Blocked Replicates}

For given $p$ and $M<M_{0}$ the class $\mathcal{D}_{M}^{p}$ is empty. However, designs in $M$ blocked replicates can be constructed allowing estimation of all main effects and of a subset of interactions. 


\subsection{Designs to Maximise Estimability for $M<M_{0}$}

For a $p, M$ combination with $M<M_{0}$, a generator matrix comprising columns from $X_{M}$ necessarily involves column duplication and gives a design in $M$ blocked replicates from which all main effects but only a subset of interactions are estimable. The number of factors can be expressed uniquely as $p=\alpha\left(2^{M}-1\right)+\beta$ where $\alpha=\left[p /\left(2^{M}-1\right)\right]$ and $\beta$, the remainder, takes some integer value in $\left[0,2^{M}-2\right]$. An upper bound, $\phi$, for the number of pairs of distinct columns in a set of $p$ columns from $X_{M}$ is obtained by manipulation of Binomial coefficients:

$$
\phi=\left(\begin{array}{l}
p \\
2
\end{array}\right)-\alpha \beta-\left(2^{M}-1\right)\left(\begin{array}{l}
\alpha \\
2
\end{array}\right) .
$$

This is also an upper bound for the number of estimable interactions from $M$ blocked $2^{p}$ replicates and reduces to $\left(\begin{array}{l}p \\ 2\end{array}\right)$ when $M \geq M_{0}$. The bound of (5.1) prompts an alternative to Theorem 1, which accommodates designs with $M<M_{0}$.

Theorem 2. A design in $M<M_{0}$ blocked $2^{p}$ replicates enables estimation of all main effects and of $\phi$ interactions iff the generator matrix comprises $\alpha+1$ copies of each of $\beta$ columns of $X_{M}$, and $\alpha$ copies of each of the remaining $2^{M}-\beta-1$ columns.

The proof of Theorem 2 is along the same lines as that of Theorem 1 and is omitted. For a design satisfying Theorem 2 , the non-estimable interactions are those corresponding to a pair of identical columns in the generating matrix. Note that if $M=1$, the only generator matrix satisfying Theorem 2 is $(1, \ldots, 1)$ and the single blocked replicate gives estimates of all main effects but of no interactions.

To demonstrate Theorem 2, consider $p=5, M=2$. For this combination, $\alpha=1, \beta=2$ and $\phi=8$. An example of a design in two blocked $2^{5}$ replicates which satisfies Theorem 2 , and so provides estimates of all main effects and achieves the upper bound of $\phi=8$ estimable interactions, is $\mathcal{D} 8$ with generator matrix:

$$
X^{\mathcal{D} 8}=\left(\begin{array}{lllll}
1 & 1 & 1 & 0 & 0 \\
1 & 0 & 0 & 1 & 1
\end{array}\right) .
$$

For $\mathcal{D} 8$ the non-estimable interactions are $B C$ and $D E$. 
There are experimental situations in which a bespoke design enabling estimation of fewer than $\phi$ interactions is preferable to one satisfying Theorem 2. The next example demonstrates use of the generator matrix design construction approach to tailor a design to a specific problem.

\subsection{Ceramic Example}

A manufacturing experiment is to be conducted to investigate the effects of five twolevel factors on the quality of ceramic pots. The factors are $A$ : clay purity, $B$ : particle size, $C$ :drying time, $D$ : mixing method and $E$ :compaction pressure. The kiln capacity allows items to be fired in pairs. Two kilns are available. Thus, a design comprising two blocked $2^{5}$ replicates is appropriate, with one replicate per kiln and blocks relating to individual kiln firings. From knowledge of the process, it is believed that interactions between any pair of $C, D, E$ can be considered negligible. All main effects and all interactions involving $A$ and $B$ are of interest. Use of $\mathcal{D} 8$ would give estimates of all main effects and maximise the number of estimable interactions. However no estimate would be given for $B C$. Further, no rearrangement of $X^{\mathcal{D} 8}$ gives a design enabling estimation of all interactions of interest. Consider instead $\mathcal{D} 9$ with generator matrix:

$$
X^{\mathcal{D} 9}=\left(\begin{array}{lllll}
1 & 0 & 1 & 1 & 1 \\
0 & 1 & 1 & 1 & 1
\end{array}\right) .
$$

This design provides estimates of all main effects but of only six interactions, two fewer than $\mathcal{D} 8$. However, unlike $\mathcal{D} 8$ it does enable estimation of all interactions of interest, since only $C D, C E$ and $D E$ are inestimable.

\section{Construction of Designs in Blocked $2^{p-r}$ Fractional Replicates}

The DCCP of $\S 3$ yields all designs in $\mathcal{D}_{M}^{p}$ for given $M, p$ with $M \geq M_{0}$. In many cases it will be unrealistic to use designs in blocked $2^{p}$ replicates due to the number of runs: for example designs in $\mathcal{D}_{4}^{p}$ with $p \geq 8$ involve in excess of 1,000 runs. However, members of 
$\mathcal{D}_{M}^{p}$ can be used as root designs for designs in fractional blocked replicates. An example is now given to illustrate the structure of such a design.

\subsection{Example of a Design in Blocked $2^{5-1}$ Fractional Replicates}

First, consider a blocked $2^{5}$ replicate with $\mathrm{g}_{1}=a c$. This yields estimates of $A, C$ and $A B, A D, A E, B C, C D, C E$. Next consider a half replicate of the $2^{5}$ factorial with fraction generator $B D E$. Treatment combinations in the principal fraction are those with an even

number of $B, D, E$ at high level. These are contained in the eight blocks of the blocked $2^{5}$ replicate displayed below.

\begin{tabular}{|c|c|c|c|c|c|c|c|}
\hline$(1)$ & $a$ & $b d$ & $a b d$ & $b e$ & $a b e$ & $d e$ & ade \\
$a c$ & $c$ & $a b c d$ & $b c d$ & $a b c e$ & $b c e$ & acde & $c d e$ \\
\hline
\end{tabular}

In the half replicate, $A, C$ have four-factor interaction aliases and $A B, A D, A E, B C, C D, C E$ have three-factor interaction aliases. Thus, the effects of interest estimable from the blocked $2^{5}$ replicate are also estimable from the eight blocks of (6.1), which is termed a blocked $2^{5-1}$ replicate.

Now let $\mathcal{D} 10$ be the design in $\mathcal{D}_{4}^{5}$ with generator matrix:

$$
X_{\mathcal{D} 10}=\left(\begin{array}{lllll}
1 & 0 & 1 & 0 & 0 \\
1 & 1 & 1 & 1 & 0 \\
1 & 1 & 1 & 0 & 1 \\
1 & 1 & 0 & 1 & 1
\end{array}\right)
$$

From the rows of $X_{\mathcal{D} 10}$ the replicate generators are $\mathrm{g}_{1}=a c, \mathrm{~g}_{2}=a b c d, \mathrm{~g}_{3}=a b c e$, $\mathrm{g}_{4}=a b d e$. Design $\mathcal{D} 10$ can be used as the root for another design, $\mathcal{D} 11$ say, in four blocked $2^{5-1}$ replicates. This is achieved by selecting a suitable fraction generator for each replicate of $\mathcal{D} 10$. Using fraction generators $B D E, A B C D, A B C E$ and $A B D E$ for replicates one to 
Table 4: Estimates from $\mathcal{D} 10$ and $\mathcal{D} 11$

\begin{tabular}{cl}
$\begin{array}{c}\text { blocked replicate } \\
\text { or half replicate }\end{array}$ & estimable effects \\
\hline 1 & $A, C, A B, A D, A E, B C, C D, C E$ \\
2 & $A, B, C, D, A E, B E, C E, D E$ \\
3 & $A, B, C, E, A D, B D, C D, D E$ \\
4 & $A, B, D, E, A C, B C, C D, C E$ \\
\hline
\end{tabular}

\begin{tabular}{cccccccccc}
\multicolumn{1}{l}{ Table 5: Estimation summary for $\mathcal{D} 10$ and $\mathcal{D} 11$} \\
\hline$N$ & $n_{1}$ & $n_{2}$ & $n_{3}$ & $n_{4}$ & $T$ & $t_{1}$ & $t_{2}$ & $t_{3}$ & $t_{4}$ \\
\hline 14 & 0 & 2 & 2 & 1 & 18 & 4 & 4 & 2 & 0 \\
\hline
\end{tabular}

four gives the half replicates displayed in (6.1) and (6.2)-(6.4).

\begin{tabular}{|c|c|c|c|c|c|c|c|}
\hline $\begin{array}{c}(1) \\
a b c d\end{array}$ & $a b$ & $a c$ & $a d$ & $a b e$ & $a c e$ & $a d e$ & $e$ \\
& $b d$ & $b c$ & $c d e$ & $b d e$ & $b c e$ & $a b c d e$ \\
\hline$(1)$ & $a b$ & $a c$ & $a e$ & $a b d$ & $a c d$ & $a d e$ & $d$ \\
$a b c e$ & $c e$ & $b e$ & $b c$ & $c d e$ & $b d e$ & $b c d$ & $a b c d e$ \\
\hline$(1)$ & $a b$ & $a d$ & $a e$ & $a b c$ & $a c d$ & $a c e$ & $c$ \\
$a b d e$ & $d e$ & $b e$ & $b d$ & $c d e$ & $b c e$ & $b c d$ & $a b c d e$ \\
\hline
\end{tabular}

The effects of interest estimable from each blocked $2^{5-1}$ replicate are exactly the same as from the full blocked $2^{5}$ replicate. Thus every main effect and interaction is estimable from $\mathcal{D} 11$. Table 4 gives the estimates provided by each replicate of $\mathcal{D} 10$ and the corresponding half replicate of $\mathcal{D} 11$. The estimability properties of the designs are summarised in Table 5. The designs differ in the number of runs and in the precision of estimation. Design $\mathcal{D} 11$ requires only 64 runs, compared to 128 for $\mathcal{D} 10$. For $\mathcal{D} 11$, the largest and average variance for main effect estimators are $v_{n}=0.125 \sigma^{2}$ and $\bar{v}_{n}=0.0958 \sigma^{2}$ respectively. For the interactions, the largest and average variances are $v_{t}=0.25 \sigma^{2}$ and $\bar{v}_{t}=0.1667 \sigma^{2}$. These are twice as large as the values for $\mathcal{D} 10$, but in most cases it would be expected that the saving in resources afforded by use of $\mathcal{D} 11$ make the latter a more appealing design. 


\subsection{Construction of Blocked $2^{p-r}$ Replicates}

For each replicate of $\mathcal{D} 10$ in $\S 6.1$, the fraction generator used to determine the corresponding half replicate of $\mathcal{D} 11$ is such that the treatment combinations of the half replicate are exactly contained in half the blocks of the full replicate. Further, effects of interest estimable in each full replicate are also estimable from the blocked $2^{5-1}$ replicate. In general, a blocked $2^{p-r}$ replicate will be taken to mean a $1 / 2^{r}$ fraction of the blocks of a blocked $2^{p}$ replicate, including the principal block, from which the same main effects and interactions are estimable as from the full blocked replicate. A blocked $2^{p-r}$ replicate is completely specified by a replicate generator and $r$ independent fraction generators.

Given a replicate generator, the fraction generators are chosen so the treatment combinations in the fractional replicate are precisely those in a subset of the blocks of the blocked replicate, and so that effects of interest are not compromised in the fractional replicate. Use of resolution $\mathrm{V}$ fractional replicates would ensure no aliasing between effects of interest. However, since only $q$ main effects and $q(p-q)$ interactions are estimated from a blocked $2^{p}$ replicate, a less stringent resolution requirement will ensure preservation of these effects in a fractional replicate. This is observed in $§ 6.1$ where use of three- and four-factor interactions as fraction generators preserved estimable effects of interest.

Let the set of selected factors of a replicate generator be $\mathcal{G}=\left\{G_{1}, \ldots, G_{q}\right\}$ and the set of remaining factors be $\mathcal{H}=\left\{H_{1}, \ldots, H_{p-q}\right\}$. The blocked $2^{p}$ replicate provides estimates of the main effects $G_{i}$ and interactions $G_{i} H_{j}$, with $G_{i} \in \mathcal{G}, H_{j} \in \mathcal{H}$. Conditions on the $r$ fraction generators to yield a blocked $2^{p-r}$ replicate are given by the following result:

Theorem 3. A blocked $2^{p}$ replicate with replicate generator g yields a blocked $2^{p-r}$ replicate if the $r$ fraction generators are such that:

(i) Every fraction generator has an even number of factors in common with $\mathcal{G}$;

(ii) Every fraction generator and generalized interaction between fraction generators contains at least four factors from $\mathcal{G}$ or at least three factors from $\mathcal{H}$, or both.

Proof: All blocks of the blocked $2^{p}$ replicate are cosets of $\{1, \mathrm{~g}\}$. If (i) is satisfied then the principal $2^{p-r}$ fraction specified by the $r$ fraction generators contains the treatment 
combination $g$, and the treatment combinations of the principal fraction are exactly those contained in $2^{p-r-1}$ blocks of the blocked $2^{p}$ replicate. By (ii), aliases of $G_{i}$ and of $G_{i} H_{j}$ in the fractional replicate are interactions between three or more factors, for all $G_{i} \in \mathcal{G}, H_{j} \in$ $\mathcal{H}$ and are negligible. The result follows.

Given g, a blocked $2^{p-r}$ replicate is obtained by the same principles used to form fractional factorials of resolutions IV and III. The process involves identification of $r=$ $r_{1}+r_{2}$ fraction generators, where $r_{1}, r_{2} \geq 0$. If $r_{1}>0$ then $r_{1}$ fraction generators are required for a $2^{q-r_{1}}$ fraction in factors in $\mathcal{G}$ that is resolution IV or better. Likewise, if $r_{2}>0$, then $r_{2}$ fraction generators are required for a $2^{p-q-r_{2}}$ fraction in factors of $\mathcal{H}$ of resolution III or better. The $r$ fraction generators together yield a blocked $2^{p-r}$ replicate.

Table 6 lists fraction generators, obtained using the approach of Box and Hunter (1961). The left hand side of the table gives fraction generators corresponding to the construction of resolution IV $2^{q-r_{1}}$ designs in factors of $\mathcal{G}$ for $q \leq 15$, and the right hand side gives fraction generators corresponding to resolution III $2^{p-q-r_{2}}$ designs in factors of $\mathcal{H}$, for $p-q \leq 15$. For each $q$ and $p-q$, the largest possible values of $r_{1}$ and $r_{2}$ are given as $r_{1}\langle q\rangle$ and $r_{2}\langle p-q\rangle$ respectively. For a blocked $2^{p}$ replicate with a replicate generator of length $q$, Table 6 provides $r_{1}\langle q\rangle$ fraction generators from $\mathcal{G}$ and $r_{2}\langle p-q\rangle$ fraction generators from $\mathcal{H}$.

Use of Table 6 is explained briefly. Consider a blocked $2^{p_{*}}$ replicate with replicate generator of length $q_{*}$. If $q_{*}<4$ then $r_{1}\left\langle q_{*}\right\rangle=0$. For $q_{*}=4$ there is one fraction generator from $\mathcal{G}$, namely $G_{1} G_{2} G_{3} G_{4}$. For $5 \leq q_{*} \leq 8$, rows $q=5, \ldots, q_{*}$ give $r_{1}\left\langle q_{*}\right\rangle=q_{*}-4$ available fraction generators from $\mathcal{G}$, and for $9 \leq q_{*} \leq 15$, there are $r_{1}\left\langle q_{*}\right\rangle=q_{*}-5$ fraction generators from rows $q=9, \ldots, q_{*}$. For example, with $q_{*}=11$ six fraction generators are available: $G_{1} G_{2} G_{3} G_{6}, G_{1} G_{2} G_{4} G_{7}, G_{1} G_{2} G_{5} G_{8}, G_{1} G_{3} G_{4} G_{9}, G_{1} G_{3} G_{5} G_{10}, G_{1} G_{4} G_{5} G_{11}$. Similarly, for $p_{*}-q_{*}=3$ there is $r_{2}\langle 3\rangle=1$ fraction generator from $\mathcal{H}$. For $4 \leq p_{*}-q_{*} \leq 7$, there are $r_{2}\left\langle p_{*}-q_{*}\right\rangle=p_{*}-q_{*}-3$ fraction generators, from rows $p-q=4, \ldots, p_{*}-q_{*}$. For $8 \leq p_{*}-q_{*} \leq 15$, there are $r_{2}\left\langle p_{*}-q_{*}\right\rangle=p_{*}-q_{*}-4$ fraction generators that can be used, from rows $p-q=8, \ldots, p_{*}-q_{*}$. For example, with $p_{*}-q_{*}=5$ the fraction generators $H_{1} H_{2} H_{4}$ 
Table 6: Fraction Generators for Blocked $2^{p-r}$ Replicates.

\begin{tabular}{cclccl}
\hline$q$ & $r_{1}\langle q\rangle$ & generators & $p-q$ & $r_{2}\langle p-q\rangle$ & generators \\
\hline 0 & - & - & 0 & 0 & - \\
1 & 0 & - & 1 & 0 & - \\
2 & 0 & - & 2 & 0 & - \\
3 & 0 & - & 3 & 1 & $H_{1} H_{2} H_{3}$ \\
4 & 1 & $G_{1} G_{2} G_{3} G_{4}$ & 4 & 1 & $H_{1} H_{2} H_{4}$ \\
\hline 5 & 1 & $G_{1} G_{2} G_{3} G_{5}$ & 5 & 2 & $H_{1} H_{3} H_{5}$ \\
6 & 2 & $G_{1} G_{2} G_{4} G_{6}$ & 6 & 3 & $H_{2} H_{3} H_{6}$ \\
7 & 3 & $G_{1} G_{3} G_{4} G_{7}$ & 7 & 4 & $H_{1} H_{2} H_{3} H_{7}$ \\
8 & 4 & $G_{2} G_{3} G_{4} G_{8}$ & 8 & 4 & $H_{1} H_{2} H_{5}, H_{1} H_{3} H_{6}$, \\
\hline 9 & 4 & $G_{1} G_{2} G_{3} G_{6}, G_{1} G_{2} G_{4} G_{7}$, & & & $H_{1} H_{4} H_{7}, H_{2} H_{3} H_{8}$ \\
& & $G_{1} G_{2} G_{5} G_{8}, G_{1} G_{3} G_{4} G_{9}$ & 9 & 5 & $H_{2} H_{4} H_{9}$ \\
10 & 5 & $G_{1} G_{3} G_{5} G_{10}$ & 10 & 6 & $H_{3} H_{4} H_{10}$ \\
11 & 6 & $G_{1} G_{4} G_{5} G_{11}$ & 11 & 7 & $H_{1} H_{2} H_{3} H_{11}$ \\
12 & 7 & $G_{2} G_{3} G_{4} G_{12}$ & 12 & 8 & $H_{1} H_{2} H_{4} H_{12}$ \\
13 & 8 & $G_{2} G_{3} G_{5} G_{13}$ & 13 & 9 & $H_{1} H_{3} H_{4} H_{13}$ \\
14 & 9 & $G_{2} G_{4} G_{5} G_{14}$ & 10 & $H_{2} H_{3} H_{4} H_{14}$ \\
15 & 10 & $G_{3} G_{4} G_{5} G_{15}$ & 15 & 11 & $H_{1} H_{2} H_{3} H_{4} H_{15}$ \\
\hline
\end{tabular}

and $H_{1} H_{3} H_{5}$ are available. For ease of reference, the values of $r^{p}\langle q\rangle=r_{1}\langle p\rangle+r_{2}\langle p-q\rangle$ for $2 \leq p \leq 15$ and $1 \leq q \leq p$ are displayed in Table 7 . The construction of blocked $2^{p-r}$ replicates from blocked $2^{p}$ replicates is now demonstrated for $p=8$.

\subsection{Construction of Blocked $2^{8-r}$ Replicates}

Consider the blocked $2^{8}$ replicate with $\mathrm{g}=a b c d f g$. This has $q=6$ and $p-q=2$. Table 7 gives $r^{8}\langle 6\rangle=2$, indicating that every blocked $2^{8}$ replicate with $q=6$ can be used to produce a blocked $2^{8-2}$ replicate. From Table $6, r_{1}\langle q\rangle=r_{1}\langle 6\rangle=2$ and $r_{2}\langle p-q\rangle=r_{2}\langle 2\rangle=0$ : the available fraction generators involve only the selected factors and are $G_{1} G_{2} G_{3} G_{5}$ and $G_{1} G_{2} G_{4} G_{6}$. Any one to one mapping from $A, B, C, D, F, G$ to $\mathcal{G}$ can be used. The obvious mapping $A: G_{1}, \ldots, G: G_{6}$ corresponds to the $2^{8-2}$ quarter replicate with fraction generators $A B C F$ and $A B D G$. The treatment combinations of the principal quarter replicate occur in 32 blocks of the blocked $2^{8}$ replicate. These blocks comprise a blocked $2^{8-2}$ replicate, which is completely specified by $\mathrm{g}=a b c d f g$ and fraction generators $A B C F$ and $A B D G$.

To form a blocked $2^{8-3}$ replicate requires $r^{8}\langle q\rangle \geq 3$. From Table 7 , only blocked 
Table 7: Values of $r^{p}\langle q\rangle$

\begin{tabular}{llllllllllllllll}
\hline & 1 & 2 & 3 & 4 & 5 & 6 & 7 & 8 & 9 & 10 & 11 & 12 & 13 & 14 & 15 \\
\hline$p=2$ & 0 & 0 & & & & & & & & & & & & & \\
$p=3$ & 0 & 0 & 0 & & & & & & & & & & & & \\
$p=4$ & 1 & 0 & 0 & 1 & & & & & & & & & & & \\
$p=5$ & 1 & 1 & 0 & 1 & 1 & & & & & & & & & & \\
$p=6$ & 2 & 1 & 1 & 1 & 1 & 2 & & & & & & & & & \\
$p=7$ & 3 & 2 & 1 & 2 & 1 & 2 & 3 & & & & & & & & \\
$p=8$ & 4 & 3 & 2 & 2 & 2 & 2 & 3 & 4 & & & & & & & \\
$p=9$ & 4 & 4 & 3 & 3 & 2 & 3 & 3 & 4 & 4 & & & & & & \\
$p=10$ & 5 & 4 & 4 & 4 & 3 & 3 & 4 & 4 & 4 & 5 & & & & & \\
$p=11$ & 6 & 5 & 4 & 5 & 4 & 4 & 4 & 5 & 4 & 5 & 6 & & & & \\
$p=12$ & 7 & 6 & 5 & 5 & 5 & 5 & 5 & 5 & 5 & 5 & 6 & 7 & & & \\
$p=13$ & 8 & 7 & 6 & 6 & 5 & 6 & 6 & 6 & 5 & 6 & 6 & 7 & 8 & & \\
$p=14$ & 9 & 8 & 7 & 7 & 6 & 6 & 7 & 7 & 6 & 6 & 7 & 7 & 8 & 9 & \\
$p=15$ & 10 & 9 & 8 & 8 & 7 & 7 & 7 & 8 & 7 & 7 & 7 & 8 & 8 & 9 & 10 \\
\hline
\end{tabular}

$2^{8}$ replicates with $q \in\{1,2,7,8\}$ yield blocked $2^{8-3}$ replicates. Consider the blocked $2^{8}$ replicate with $\mathrm{g}=d e$. From Table $6, r_{1}\langle 2\rangle=0$ and $r_{2}\langle 6\rangle=3$ and a set of fraction generators is $H_{1} H_{2} H_{4}, H_{1} H_{3} H_{5}$ and $H_{2} H_{3} H_{6}$. The obvious mapping from the non-selected factors $A, B, C, F, G, H$ to $\mathcal{H}$ gives the blocked $2^{8-3}$ replicate specified by $\mathrm{g}=$ de and fraction generators $A B F, A C G$ and $B C H$.

From Table 7 it is seen that a blocked $2^{8-4}$ replicate is obtained from a blocked $2^{8}$ replicate with $q \in\{1,8\}$ and that no blocked $2^{8-r}$ replicate can be formed with $r>4$.

\subsection{Construction of Designs in Blocked $2^{p-r}$ Replicates}

To form a design in $M \geq M_{0}$ blocked $2^{p-r}$ replicates, a root design is selected from $\mathcal{D}_{M}^{p}$ with replicate generator lengths $q_{1}, \ldots, q_{M}$. The value of $r$ is chosen with $r \leq$ $\min _{i \in\{1, \ldots, M\}}\left\{r^{p}\left\langle q_{i}\right\rangle\right\}$. For each replicate of the root, $r$ fraction generators are selected using Table 6, to produce the $M$ blocked $2^{p-r}$ replicates of the final design. For given $p, r, M$, the class of such designs will be denoted by $\mathcal{D}_{M}^{p-r}$. Thus $\mathcal{D} 11$ of $\S 6.1$ is contained in $\mathcal{D}_{4}^{5-1}$.

A design in $\mathcal{D}_{M}^{p-r}$ has $M \times 2^{p-r}$ runs. The coefficients of observations for an estimator from a blocked $2^{p-r}$ replicate are $\pm 1\left(2^{p-r-1}\right)^{-1}$ and the variance is $\sigma^{2}\left(2^{p-r-2}\right)^{-1}$. An effect 
that is estimable from $n$ fractional replicates has an estimator which is the average of the $n$ separate estimators and has variance $\sigma^{2}\left(n 2^{p-r-2}\right)^{-1}$. The quality of estimation of a design can be summarised by the largest and average variance of main effect estimators, denoted $v_{n}$ and $\bar{v}_{n}$, and the largest and average variances of interaction estimates, denoted $v_{t}$ and $\bar{v}_{t}$, as used in $\S 6.1$.

\section{Fractional Blocked Designs}

The approach for constructing a design in $\mathcal{D}_{M}^{p-r}$, summarised in $\S 6.4$, has selection of a root design from $\mathcal{D}_{M}^{p}$ as the starting point. The value of $r$ depends on the root design selected. To provide guidance on available $p, r, M$ combinations and on selection of the root design, designs in $\mathcal{D}_{M}^{p-r}$ are proposed for $4 \leq p \leq 15$.

\subsection{Criteria for Design Selection}

A design will be reported from a non-empty $\mathcal{D}_{M}^{p-r}$ if there are no designs in $M-1$ blocked $2^{p-r}$ replicates or in $M$ blocked $2^{p-r-1}$ replicates, that is, if $\mathcal{D}_{M}^{p-r-1}$ and $\mathcal{D}_{M-1}^{p-r}$ are empty. The design given from a class will be that with highest ranked root design according to $§ 3.5$. This is equivalent to selecting the highest ranked design in $\mathcal{D}_{M}^{p-r}$, by the same ranking. For each $p$, designs from up to four classes are reported. For two designs with parameters $p, r_{A}, M_{A}$ and $p, r_{B}, M_{B}$, with $r_{A}>r_{B}$ and $M_{A}>M_{B}$, no design ranking is suggested because no method will be appropriate in all situations. In some experiments minimising the total number of runs or the number of runs per fractional replicate will be of prime importance, whereas in others the quality of estimation will be fundamental. The number of runs and $v_{n}, \bar{v}_{n}, v_{t}$ and $\bar{v}_{t}$ are reported for each design. For illustration, $p=8$ is considered now in detail.

\subsection{Designs for $\boldsymbol{p}=\mathbf{8}$}

For $p=8$, at least four blocked replicates are required to estimate all main effects and interactions. Thus, the first class of interest has form $\mathcal{D}_{4}^{8-r}$, where $r$ is as large as possible. From Table $7, r^{8}\langle q\rangle \geq 2$. Hence, for every blocked $2^{8}$ replicate, two fraction generators 
can be found to yield a blocked $2^{8-2}$ replicate. Thus every member of $\mathcal{D}_{4}^{8}$ is the root for a design in $\mathcal{D}_{4}^{8-2}$. From Table $7, r^{8}\langle q\rangle \geq 3$ requires $q \in\{1,2,7,8\}$, and so to construct a design in $\mathcal{D}_{4}^{8-3}$ requires a root in $\mathcal{D}_{4}^{8}$ with all $q_{i}$ in $\{1,2,7,8\}$. No design of $\mathcal{D}_{4}^{8}$ has this property and consequently $\mathcal{D}_{4}^{8-3}$ is empty. Backtracking, $\mathcal{D}_{4}^{8-2}$ is the first class of interest. The highest ranking design from Table A.12 is used as the root. The replicate generators are given below, in each case with two fraction generators found from Table 6 . Note that the first fractional replicate was encountered in $\S 6.3$. The resulting design, $\mathcal{D} 12$, in $\mathcal{D}_{4}^{8-2}$ has 256 runs.

\begin{tabular}{cc}
\hline \multicolumn{2}{c}{$\mathcal{D} 12$ in $\mathcal{D}_{4}^{8-2}$} \\
replicate generator & fraction generators \\
\hline abcdfg & $A B D G, A B C F$ \\
abcefh & $A B E H, A B C F$ \\
abdeg & $A B E G, C F H$ \\
acdeh & $A C E H, B F G$ \\
\hline
\end{tabular}

To find the next class of interest, the smallest $M$ for which $\mathcal{D}_{M}^{8-r}$ is non-empty for $r>2$ is identified. From Table 7 , a design in $\mathcal{D}_{5}^{8-3}$ requires a root in $\mathcal{D}_{5}^{8}$ with all $q_{i}$ in $\{1,2,7,8\}$. There is no such root design and so $\mathcal{D}_{5}^{8-3}$ is empty. Several designs in $\mathcal{D}_{6}^{8}$ have all six $q_{i}$ in $\{1,2,7,8\}$, but none have all $q_{i}$ in $\{1,8\}$. Therefore, $\mathcal{D}_{6}^{8-3}$ is the next class of note. Design $\mathcal{D} 13$ has 192 runs and uses the highest ranked design in $\mathcal{D}_{6}^{8}$ with the required property as root. The replicate generators are given below, each with three fraction generators. Note that the third replicate was covered in $§ 6.3$. 


\begin{tabular}{cc}
\hline \multicolumn{2}{c}{$\mathcal{D} 13$ in $\mathcal{D}_{6}^{8-3}$} \\
replicate generator & fraction generators \\
\hline abdefgh & $A B D F, A B E G, A D E H$ \\
acdefgh & $A C D F, A C E G, A D E H$ \\
$d e$ & $A B F, A C G, B C H$ \\
$d f$ & $A B E, A C G, B C H$ \\
abcdefg & $A B C E, A B D F, A C D G$ \\
abcdefh & $A B C E, A B D F, A C D H$ \\
\hline
\end{tabular}

No designs in $\mathcal{D}_{7}^{8}$ have all $q_{i}$ in $\{1,8\}$ but two designs in $\mathcal{D}_{8}^{8}$ have this property. Design $\mathcal{D} 14$ in $\mathcal{D}_{8}^{8-4}$ has the highest ranked of these as root. The replicate generators and fraction generators of the 128 run design are:

\begin{tabular}{|c|c|}
\hline \multicolumn{2}{|r|}{$\mathcal{D} 14$ in $\mathcal{D}_{8}^{8-4}$} \\
\hline replicate generator & fraction generators \\
\hline$a b c d e f g h$ & $A B C E, A B D F, A C D G, B C D H$ \\
\hline$a$ & $B C E, B D F, C D G, B C D H$ \\
\hline$b$ & $A C E, A D F, C D G, A C D H$ \\
\hline$c$ & $A B E, A D F, B D G, A B D H$ \\
\hline$d$ & $A B E, A C F, B C G, A B C H$ \\
\hline$e$ & $A B D, A C F, B C G, A B C H$ \\
\hline$f$ & $A B D, A C E, B C G, A B C H$ \\
\hline$g$ & $A B D, A C E, B C F, A B C H$ \\
\hline
\end{tabular}

All three designs make significant resource savings on designs in $\mathcal{D}_{4}^{8}$. From Table 8, the variance measures of $\mathcal{D} 13$ in $\mathcal{D}_{6}^{8-3}$, with 192 runs, come close to those of the 256 run design, $\mathcal{D} 12$, in $\mathcal{D}_{4}^{8-2}$. The variance measures of $\mathcal{D} 14$ in $\mathcal{D}_{8}^{8-4}$, with 128 runs, are considerably larger.

\subsection{Injection Moulding Process Example}

The process of injection moulding involves many factors and fractional factorial experiments have been used to improve the quality of items manufactured in this way. See, for 
example Ghose et al. (2015). Consider a factory which manufactures auto components by injection moulding. An experiment is planned to explore the effects of eight two-level factors on the surface appearance of the components. The factors are A: injection pressure, $B$ :ram speed, $C$ : clamp force, $D$ : barrel temperature, $E$ : nozzle temperature, $F$ : cooling time, $G$ : gas injection method and $H$ : holding pressure. Eight injection moulding machines are available. Due to the time needed to set up the process with the factors at specific levels, two runs can be carried out in a machine in one shift. The model of (2.1) applies, with machines as replicates and blocks as two runs in the same machine and same shift. Any of the designs in $\S 7.2$ can be used. Which design is most appropriate depends on the importance of balancing various aspects of the designs including: the total number of runs; the number of runs per replicate; the quality of the estimators. For example $\mathcal{D} 14$, with eight fractional replicates of 16 runs would lead tio an experiment that would be concluded most quickly, whereas $\mathcal{D} 13$, with six fractional replicates of 32 runs would take longer to conduct but would give more precise estimators.

\subsection{Standard Designs}

Inspection of Table 7 indicates that for $4 \leq p \leq 15$, except for $p=5,9$, a root design with all $q_{i} \in S_{1}=\{1, p\}$ enables construction of designs in blocked $2^{p-r}$ replicates with larger $r$ than is otherwise possible. For $p=5,8,9$ and $12, \ldots, 15$ there is a similar but (except for $p=5,9)$ lesser advantage in using a root design with all $q_{i} \in S_{2}=\{1,2, p-1, p\}$.

No design in $\mathcal{D}_{M}^{p}$ with $M<p$ has all $q_{i} \in S_{1}$, but $\mathcal{D}_{p}^{p}$ contains two such designs. A Type 1 design is a member of $\mathcal{D}_{p}^{p-r}$, where $r=r^{p}\langle 1\rangle$, with root the highest ranked design of $\mathcal{D}_{p}^{p}$ having all $q_{i} \in S_{1}$. The Type 1 designs are generated by Method 1 of Jacroux (2010), by a different approach. Likewise, some members of $\mathcal{D}_{p-s}^{p}$, where $s=[p / 3]$, have all $q_{i}$ in $S_{2}$ and no $\mathcal{D}_{M}^{p}$ with $M<p-s$ contains any such design. For $p \geq 4$, a Type 2 design is a member of $\mathcal{D}_{p-s}^{p-r}$, where $r=r^{p}\langle 2\rangle$, with root the highest ranked member of $\mathcal{D}_{p-s}^{p}$ with all $q_{i} \in S_{2}$.

For $p=8$, the designs of $\S 7.2$ in $\mathcal{D}_{8}^{8-4}$ and $\mathcal{D}_{6}^{8-3}$ are Type 1 and 2 respectively. Generator matrices for root designs yielding Type 1 and Type 2 designs are now given for general $p$, 
with summary properties of the resulting fractional designs.

Type 1 Design The root design is in $\mathcal{D}_{p}^{p}$ and has generator matrix:

$$
X_{\mathcal{D}}=\left(\begin{array}{c|c}
1_{p-1}^{T} & 1 \\
\hline I_{p-1} & 0
\end{array}\right),
$$

where $I_{y}$ is the $y \times y$ identity matrix. The estimator summary for a Type 1 design is:

\begin{tabular}{cccccc}
\hline$N$ & $n_{1}$ & $n_{2}$ & $T$ & $t_{1}$ & $t_{2}$ \\
\hline $2 p-1$ & 1 & $p-1$ & $(p-1)^{2}$ & $p-1$ & $(p-1)(p-2) / 2$ \\
\hline
\end{tabular}

The variance summaries for main effects and interactions are $v_{n}=\sigma^{2} / 2^{p-r-2}, \bar{v}_{n}=\sigma^{2}(p+$ 1) $/ p 2^{p-r-1}, v_{t}=\sigma^{2} / 2^{p-r-2}$ and $\bar{v}_{t}=\sigma^{2}(p+2) / p 2^{p-r-1}$, respectively.

Type 2 Design There are three cases, depending on the residue of $p$ modulo 3 . In each case the root design is in $\mathcal{D}_{p-s}^{p}$. The notation $\otimes$ denotes the Kronecker product.

$p=3 s$

$$
X_{\mathcal{D}}=\left(\frac{\left(\begin{array}{lll}
1 & 1 & 0 \\
1 & 0 & 1
\end{array}\right)}{1_{s-1} \otimes\left(\begin{array}{lll}
0 & 0 & 0 \\
0 & 0 & 0
\end{array}\right) \mid I_{s-1}^{T} \otimes\left(\begin{array}{lll}
1 & 1 & 1 \\
1 & 1 & 1
\end{array}\right)}\right),
$$

$p=3 s+1$

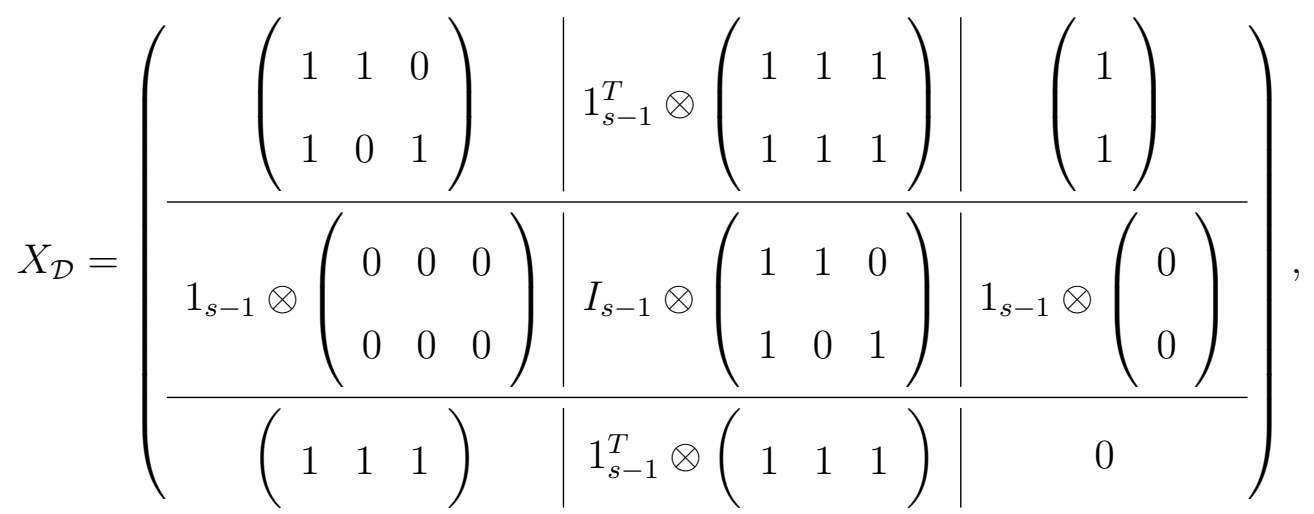


$\underline{p=3 s+2}$

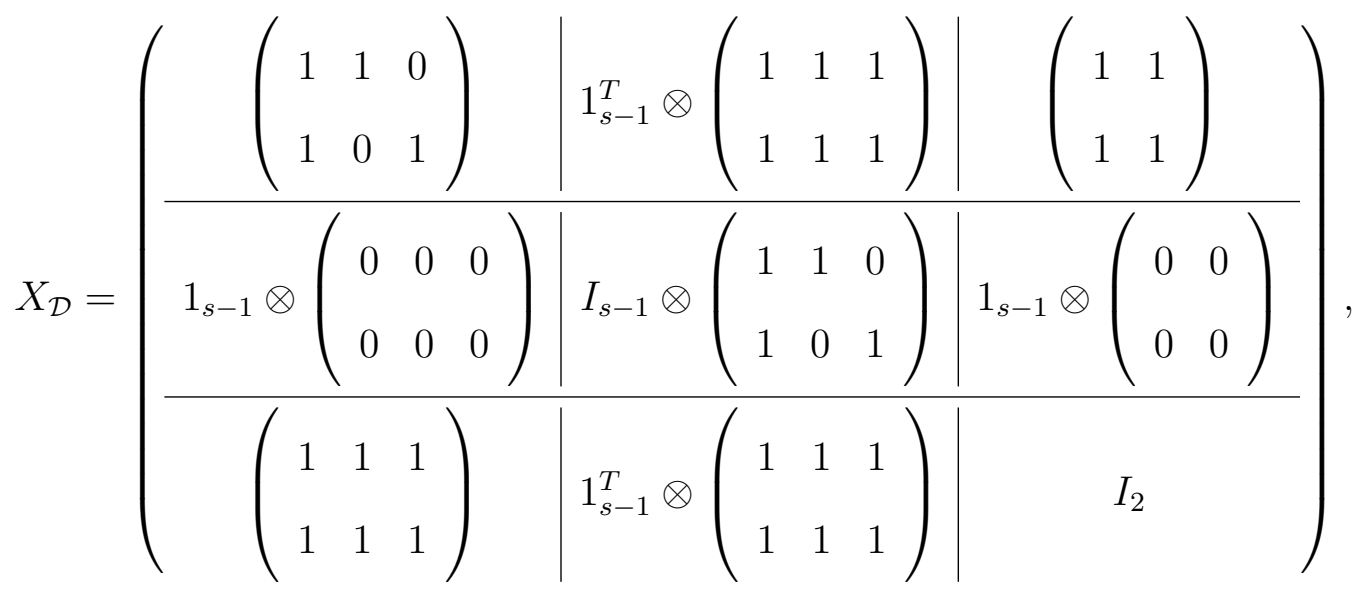

Estimability properties of Type 2 designs are summarised as follows:

\begin{tabular}{cccccccc}
\hline$p$ & $N$ & $n_{1}$ & $n_{2}$ & $n_{3}$ & $n_{4}$ & $n_{5}$ & $n_{6}$ \\
\hline $3 s$ & $10 s-6$ & 2 & 1 & $2(s-1)$ & $s-1$ & 0 & 0 \\
$3 s+1$ & $13 s-4$ & 0 & 3 & 1 & $2(s-1)$ & $s-1$ & 0 \\
$3 s+2$ & $16 s$ & 0 & 0 & 4 & 1 & $2(s-1)$ & $s-1$ \\
\hline
\end{tabular}

\begin{tabular}{cccccc}
\hline$p$ & $T$ & $t_{1}$ & $t_{2}$ & $t_{3}$ & $t_{4}$ \\
\hline $3 s$ & $12 s^{2}-14 s+6$ & $4 s-2$ & $2 s^{2}-1$ & $2 s^{2}-4 s+2$ & $\left(s^{2}-3 s+2\right) / 2$ \\
$3 s+1$ & $12 s^{2}-7 s+4$ & $4 s-1$ & $2 s^{2}+2 s-1$ & $2 s^{2}-3 s+1$ & $\left(s^{2}-3 s+2\right) / 2$ \\
$3 s+2$ & $12 s^{2}+4$ & $4 s$ & $2 s^{2}+4 s$ & $2 s^{2}-2 s$ & $\left(s^{2}-3 s+2\right) / 2$ \\
\hline
\end{tabular}

The variance measures are:

\begin{tabular}{ccccc}
\hline$p$ & $v_{n}$ & $\bar{v}_{n}$ & $v_{t}$ & $\bar{v}_{t}$ \\
\hline $3 s$ & $\frac{\sigma^{2}}{2^{3 s-r-2}}$ & $\frac{(11 s+19) \sigma^{2}}{9 s \times 2^{3 s-r}}$ & $\frac{\sigma^{2}}{2^{3 s-r-2}}$ & $\frac{\left(43 s^{2}+55 s-38\right) \sigma^{2}}{9 s(3 s-1) \times 2^{3 s-r}}$ \\
$3 s+1$ & $\frac{\sigma^{2}}{2^{3 s-r}}$ & $\frac{(21 s+34) \sigma^{2}}{15(3 s+1) 2^{3 s-r}}$ & $\frac{\sigma^{2}}{2^{3 s-r-1}}$ & $\frac{\left(43 s^{2}+87 s-22\right) \sigma^{2}}{9 s(3 s+1) 2^{3 s-r+1}}$ \\
$3 s+2$ & $\frac{\sigma^{2}}{3 \times 2^{3 s-r}}$ & $\frac{(34 s+61) \sigma^{2}}{60(3 s+2) 2^{3 s-r}}$ & $\frac{\sigma^{2}}{2^{3 s-r}}$ & $\frac{\left(43 s^{2}+119 s+6\right) \sigma^{2}}{3(3 s+2)(3 s+1) 2^{3 s-r+2}}$ \\
\hline
\end{tabular}

\subsection{Recommended Designs}

Table 8 contains designs for $4 \leq p \leq 15$ according to the criteria set out in $\S 7.1$. Due to space considerations, the fraction generators for each replicate of the given designs are not 
included. These can be obtained from Table 6. Alternatively, complete information on the fraction generators is contained in the supplementary file. The Type 1 and Type 2 designs are indicated in Table 8. For $p=5,9$ the Type 1 designs are not listed since, in these cases, the fractional replicates of the Type 1 and Type 2 designs have the same numbers of runs, but the Type 2 design has smaller $M$. Similarly, for $p=6,7,10,11$ the Type 2 designs are not listed since values of $q$ apart from those in $S_{2}$ have $r^{p}\langle q\rangle=r^{p}\langle 2\rangle$, enabling construction of designs in $\mathcal{D}_{M}^{p-r}$, with $r=r^{p}\langle 2\rangle$ and $M<p-s$. The value of $r^{4}\langle 2\rangle$ is zero and so the Type 2 design for $p=4$ does not yield a design in fractional replicates.

Generally, designs with fewer runs have higher variance measures. When choosing a design, these aspects need to be balanced given the particular experimental situation. Although no ranking is suggested for designs with common $p$ but different $r, M$, a few designs merit particular mention. For $p=6$, both designs have 96 runs. The design in $\mathcal{D}_{3}^{6-1}$ outperforms the Type 1 design in all variance measures. For $p=7$ and $p=10$, the designs in $\mathcal{D}_{3}^{7-2}$ and $\mathcal{D}_{4}^{10-4}$ have fewer runs than the corresponding Type 1 designs, but outperform these in all variance measures. For $p=13$, the design in $\mathcal{D}_{4}^{13-6}$ has fewer runs but better variance properties than the Type 2 design.

For $p \geq 12$, the Type 1 designs minimise the number of runs. These designs use the smallest fractional replicates but have the disadvantage that each fractional replicate provides only a small number of estimators with the consequence that, for given $p$, the variance summaries are higher for Type 1 designs than for other designs of Table 8.

\subsection{Comparison with other Methods}

It is informative to compare properties of Table 8 designs with those from other constructions. Table 9 gives designs for $5 \leq p \leq 8$ from Method 2 of Jacroux (2010), from the construction in $[(p+3) / 2]$ half replicates of Wang (2004) and from Yang and Draper (2003), all expressed in the format of replicate generator sets. Method 2 of Jacroux is based on Hadamard vector products. In terms of blocked fractional replicates a Method 2 design has root in $\mathcal{D}_{p-1}^{p}$ with all $q_{i}=2$, giving a design in $\mathcal{D}_{p-1}^{p-r}$ with $r=r^{p}\langle 2\rangle$. The designs have 
Table 8: Designs for $4 \leq p \leq 15$

\begin{tabular}{|c|c|c|c|c|c|c|}
\hline$p, r, M$ & Replicate Generators & Runs & $v_{n} / \sigma^{2}$ & $\bar{v}_{n} / \sigma^{2}$ & $v_{t} / \sigma^{2}$ & $\bar{v}_{t} / \sigma^{2}$ \\
\hline $4^{a}, 1,4$ & $a b c d, a, b, c$ & 32 & 0.50000 & 0.31250 & 0.50000 & 0.37500 \\
\hline $5^{b}, 1,4$ & $a b c d$, abce, abde, acde & 64 & 0.08333 & 0.07919 & 0.25000 & 0.17500 \\
\hline $6,1,3$ & $a b c e, a b d f, a c d$ & 96 & 0.12500 & 0.07813 & 0.12500 & 0.08889 \\
\hline $6^{a}, 2,6$ & abcdef $, a, b, c, d, e$ & 96 & 0.25000 & 0.14583 & 0.25000 & 0.16667 \\
\hline $7,2,3$ & $a b c e, a b d f, a c d g$ & 96 & 0.12500 & 0.08452 & 0.12500 & 0.08610 \\
\hline $7^{a}, 3,7$ & $a b c d e f g, a, b, c, d, e, f$ & 112 & 0.25000 & 0.14286 & 0.25000 & 0.16071 \\
\hline $8,2,4$ & $a b c d f g$, abce $f h$, abdeg, acdeh & 256 & 0.03125 & 0.02409 & 0.06250 & 0.03962 \\
\hline $8^{b}, 3,6$ & abdefgh, acdefgh, de, df, abcdefg, abcdefh & 192 & 0.04167 & 0.03359 & 0.12500 & 0.07738 \\
\hline $8^{a}, 4,8$ & $a b c d e f g h, a, b, c, d, e, f, g$ & 128 & 0.25000 & 0.14063 & 0.25000 & 0.15625 \\
\hline $9,3,4$ & $a b c d f g$, abce fh, abdegi, acdehi & 256 & 0.03125 & 0.02488 & 0.06250 & 0.03848 \\
\hline $9^{b}, 4,6$ & abdefghi, acdefghi, de, df, gh, gi & 192 & 0.12500 & 0.06019 & 0.12500 & 0.07436 \\
\hline $10,4,4$ & abcdefi, abcdghj, abeg, acfh & 256 & 0.06250 & 0.03385 & 0.06250 & 0.03796 \\
\hline $10^{a}, 5,10$ & $a b c d e f g h i j, a, b, c, d, e, f, g, h, i$ & 320 & 0.12500 & 0.06875 & 0.12500 & 0.07500 \\
\hline $11,4,4$ & abcdfgh, abcefij, abdegik, acdehjk & 512 & 0.01563 & 0.01302 & 0.03125 & 0.01861 \\
\hline $11,5,5$ & $\begin{array}{l}\text { abcdeghi, abcdfgjk, abcefghj, } \\
\text { abdefhik, acdefijk }\end{array}$ & 320 & 0.02083 & 0.01771 & 0.06250 & 0.03835 \\
\hline $11^{a}, 6,11$ & $a b c d e f g h i j k, a, b, c, d, e, f, g, h, i, j$ & 352 & 0.12500 & 0.06818 & 0.12500 & 0.07386 \\
\hline $12,5,4$ & $a b c d f g h l$, abcefij, abdegik, acdehjk & 512 & 0.03125 & 0.01454 & 0.03125 & 0.01839 \\
\hline $12^{b}, 6,8$ & abdefghijkl, acdefghijkl, de, df, gh,gi,jk,jl & 512 & 0.06250 & 0.02734 & 0.06250 & 0.03433 \\
\hline $12^{a}, 7,12$ & abcdefghijkl, $a, b, c, d, e, f, g, h, i, j, k$ & 384 & 0.06250 & 0.03385 & 0.06250 & 0.03646 \\
\hline $13,6,4$ & abcdfghl, abcefijm, abdegik, acdehjk & 512 & 0.03125 & 0.01583 & 0.03125 & 0.01820 \\
\hline $13^{b}, 7,9$ & $\begin{array}{l}\text { abdefghijklm, acdefghijklm, de, df, gh, gi, } \\
j k, j l, \text { abcdefghijkl }\end{array}$ & 576 & 0.03125 & 0.01891 & 0.06250 & 0.03385 \\
\hline $13^{a}, 8,13$ & abcdefghijklm, $a, b, c, d, e, f, g, h, i, j, k, l$ & 416 & 0.12500 & 0.06731 & 0.12500 & 0.07212 \\
\hline $14,7,4$ & abcdfghl, abcefijm, abdegikn, acdehjk & 512 & 0.03125 & 0.01693 & 0.03125 & 0.01803 \\
\hline $14^{b}, 8,10$ & $\begin{array}{l}\text { abdefghijklmn, acdefghijklmn, de, df, gh, } \\
\text { gi, jk, jl, abcdefghijklm, abcdefghijkln }\end{array}$ & 640 & 0.02083 & 0.01466 & 0.06250 & 0.03348 \\
\hline $14^{a}, 9,14$ & $\begin{array}{l}\text { abcdefghijklmn, } a, b, c, d, e, f, g, h, i, j, \\
k, l, m\end{array}$ & 448 & 0.12500 & 0.06696 & 0.12500 & 0.07143 \\
\hline $15,8,4$ & abcdfghl, abcefijm, abdegikn, acdehjko & 512 & 0.03125 & 0.01676 & 0.03125 & 0.01788 \\
\hline $15^{b}, 9,10$ & $\begin{array}{l}\text { abdefghijklmno, acdefghijklmno, de, df, } \\
g h, g i, j k, j l, m n, \text { mo }\end{array}$ & 640 & 0.06250 & 0.02569 & 0.06250 & 0.03254 \\
\hline $15^{a}, 10,15$ & $\begin{array}{l}\text { abcdefghijklmno, } a, b, c, d, e, f, g, h, i, j, \\
k, l, m, n\end{array}$ & 480 & 0.12500 & 0.06250 & 0.12500 & 0.06641 \\
\hline
\end{tabular}

a Type 1 design

${ }^{b}$ Type 2 design 
Table 9: Designs for $5 \leq p \leq 8$

\begin{tabular}{|c|c|c|c|c|c|c|c|}
\hline$p, r, M$ & replicate generators & runs & $v_{n} / \sigma^{2}$ & $\bar{v}_{n} / \sigma^{2}$ & $v_{t} / \sigma^{2}$ & $\bar{v}_{t} / \sigma^{2}$ & source \\
\hline $5,1,4$ & abcde, ace, ade, acd & 64 & 0.25000 & 0.11250 & 0.25000 & 0.15833 & Wang \\
\hline $5,1,4$ & $a b, b c, c d, d e$ & 64 & 0.25000 & 0.17500 & 0.25000 & 0.12708 & Jacroux Method 2 \\
\hline $5,1,4$ & abce, abde, acde, de & 64 & 0.12500 & 0.09583 & 0.25000 & 0.16667 & Yang and Draper \\
\hline $6,1,4$ & abcdef, ace, ade, acf & 128 & 0.12500 & 0.06076 & 0.12500 & 0.08333 & Wang \\
\hline $6,1,5$ & $a b, b c, c d, d e, e f$ & 160 & 0.12500 & 0.08333 & 0.12500 & 0.05625 & Jacroux Method 2 \\
\hline $7,1,5$ & $a b c d e f g$, aceg, adeg, acfg, acef & 320 & 0.06250 & 0.02485 & 0.06250 & 0.03373 & Wang \\
\hline $7,2,6$ & $a b, b c, c d, d e, e f, f g$ & 192 & 0.12500 & 0.08036 & 0.12500 & 0.05159 & Jacroux Method 2 \\
\hline $8,1,5$ & abcdefgh, aceg, adeg, acfg, aceh & 640 & 0.03125 & 0.01348 & 0.03125 & 0.01674 & Wang \\
\hline $8,3,7$ & $a b, b c, c d, d e, e f, f g, g h$ & 224 & 0.12500 & 0.07813 & 0.12500 & 0.04836 & Jacroux Method 2 \\
\hline
\end{tabular}

features in common with the Type 2 designs, which also use blocked $2^{p-r}$ replicates with $r=r^{p}\langle 2\rangle$. However, the Type 2 designs have the advantage that the weaker restriction of $q_{i} \in S_{2}$ gives designs in $p-s$ fractional replicates. For $p \geq 6$, the Method 2 designs require more runs than the Type 2 designs. The Type 2 designs have estimators of main effects with superior properties, as reflected by $v_{n}$ and $\bar{v}_{n}$, but the Method 2 designs offer slightly better interaction estimators. The number of runs for the designs of Wang (2004), for $p>5$ is significantly larger than for Table 8 designs. Of three designs in $\mathcal{D}_{4}^{5-1}$ given by Yang and Draper (2003), two are isomorphic to the Table 8 design and the third has considerably weaker properties with regards to main effect estimation. Comparisons of note follow: $\underline{p=5}$ The Table 8 and Table 9 designs all have 64 runs. The main effect estimators of the Table 8 design have superior properties. All four designs have equal $v_{t}$ and similar $\bar{v}_{t}$. $\underline{p=6}$ The Table 8 design in $\mathcal{D}_{3}^{6-1}$ has 96 runs compared with 128 and 160 runs of the Table 9 designs. Despite this, the designs have equal $v_{n}$ and $v_{t}$ and similar $\bar{v}_{n}$ and $\bar{v}_{t}$. $\underline{p=7}$ The design in $\mathcal{D}_{3}^{7-2}$ of Table 8 has only 96 runs compared with the 192 runs required for the Jacroux Method 2 design, which is the smallest design of Table 9 for $p=7$. Despite the difference in size, the designs have equal $v_{n}$ and $v_{t}$ values and similar $\bar{v}_{n}$ and $\bar{v}_{t}$.

$\underline{p=8}$ The design in $\mathcal{D}_{6}^{8-3}$ of Table 8 , has fewer runs than the smallest design of Table 9 for $p=8$. The Table 8 design outperforms the 224 run design with main effects estimation and has similar properties regarding estimation of interactions. 


\subsection{Designs in Fewer than $M_{0}$ Blocked Fractional Replicates}

In $\S 5$ guidance was given on the construction of designs in $M<M_{0}$ blocked replicates to estimate all main effects and a subset of interactions. Using the approach of $\S 6.4$ such designs can be used as roots for designs in $M$ blocked fractional replicates, with each fractional replicate providing the same estimates as the full replicate. For example, $\mathcal{D} 9$ of $\S 5.2$ comprises two blocked $2^{5}$ replicates and yields estimates of all main effects and six interactions. The design has $\min \left\{r^{5}\left\langle q_{1}\right\rangle, r^{5}\left\langle q_{2}\right\rangle\right\}=1$, indicating that it can take the role of root for a design in two blocked $2^{5-1}$ replicates from which all main effects and the same six interactions are estimable.

\section{Discussion}

With all designs in $\mathcal{D}_{M}^{p}$, where $M \geq M_{0}$, available as potential root designs via the DCCP, the method of $\S 7.1$ will always provide a design in blocked fractional replicates with the minimum number of runs to estimate all main effects and interactions. In $\S 7.6$ the approach is shown to encompass a number of other methods. Where only a subset of interactions are of interest, root designs obtained by the method of $\S 5$ can be used to construct designs in $M<M_{0}$ fractional replicates.

The model given in (2.1) and used throughout incorporates replicates. If the replicate term is omitted, then designs can be obtained in fewer runs. However, there are several motivations for inclusion of replicates in the model. First, replicates may relate to some practical feature of the experiment, such as days or sites. Second, the process of obtaining estimates of effects within replicates and of combining these affords the experiment a greater degree of robustness, in the event of observation loss or premature termination of the experiment, than will otherwise be the case. For example, for four factors and blocks of size two but no replicate term, JMP13 gives designs with the default number of blocks as thirteen. With 26 runs, such a design uses fewer resources than the design in $\mathcal{D}_{4}^{4-1}$ of Table 8 which has 32 runs. Should a block be lost from a design generated without 
replicates or should the experiment be ended prematurely, then many effects of interest could be non-estimable. However, in the event that data are not obtained from a fractional

replicate of the design in $\mathcal{D}_{4}^{4-1}$ of Table 8 , then the impact is limited: non-estimable effects from the damaged design will be restricted to those which are only estimable from the damaged replicate. Thirdly, the generator matrix construction approach of the work, which necessarily builds up a design in full or fractional replicates has an appealing transparency regarding the estimability properties of the design and enables the practitioner to formulate the design to accommodate the aims of the experiment.

With the exception of the standard designs in $\S 7.4$, designs are restricted to those with $p \leq 15$. For $p>15$, the task of generating complete design classes via the DCCP can be challenging. For example, consider $M_{0}=5$, which corresponds to $16 \leq p \leq 31$. For the smaller values of $p$ in this range, the large number of selections of $p$ columns from the 31 columns of $X_{5}$ makes it computationally challenging to identify every design in $\mathcal{D}_{5}^{p}$ without including Type II isomorphisms.

\section{References}

[1] Bisgaard, S. (1994) Blocking generators for small $2^{k-p}$ designs. Journal of Quality Technology 26, 288-296.

[2] Box, G.E.P., Hunter, J.S. (1961) The $2^{k-p}$ Fractional Factorial Designs Part I. Technometrics 3, 311-351.

[3] Cheng, C.S., Li, W., Ye, K. (2004) Blocked non-regular two-level factorial designs. Technometrics 46, 269-279.

[4] Draper, N.R., Guttman, I. (1997). Two-level factorial and fractional factorial designs in blocks of size two. Journal of Quality Technology 29, 71-75.

[5] Ghose, A., Montero, M. and Odell, D. (2015). Characterization of an injection mold- 
ing process for improved part quality. Technical report: Berkeley Manufacturing Institute,Department of Mechanical Engineering, University of California.

[6] Jacroux, M. (2009). Blocking in two-level non-regular fractional factorial designs. Journal of Statistical Planning and Inference 139, 1215-1220.

[7] Jacroux, M. (2010). Two-level fractional factorial designs in blocks of size two for the orthogonal estimation of all main effects and two-factor interactions. Statistics and Probability Letters 80, 926-931.

[8] Kerr, K.F. (2006). Efficient $2^{k}$ factorial designs for blocks of size 2 with microarray applications. Journal of Quality Technology 38, 309-318.

[9] Montgomery, D.C. (2012). Design and Analysis of Experiments. (eigth edition), Wiley, New York.

[10] Wang, P.C. (2004). Designing two-level fractional factorial experiments in blocks of size two. Sankhya 66, 327-342.

[11] Wang, P.C. (2007). Planning experiments when some specified interactions are investigated. Computational Statistics and Data Analysis 514143 - 4151.

[12] Wang, P.C., Cook, R.D. (2012). Analysis and efficient $2^{k-1}$ designs for experiments in blocks of size two. Quality and Reliability Engineering International 28 105-113.

[13] Yang, J.Y., Draper, N.R. (2003). Two-level factorial and fractional factorial designs in blocks of size two. Journal of Quality Technology, 35, 294-305.

\section{Appendix A. Design Classes $\mathcal{D}_{M_{0}}^{p}$ for $4 \leq p \leq 15$}


Table A.10: $p=4$, designs in $\mathcal{D}_{3}^{4}$

\begin{tabular}{lccccccccc}
\hline \multicolumn{10}{c}{$p=4$, designs have 48 runs } \\
\hline $\mathrm{rg}^{a}$ sets & $N$ & $n_{1}$ & $n_{2}$ & $n_{3}$ & $T$ & $t_{1}$ & $t_{2}$ & $t_{3}$ & ranking \\
\hline$a b c, a b d, a c d$ & 9 & 0 & 3 & 1 & 9 & 3 & 3 & 0 & 1 \\
$a b c, a b d, a c$ & 8 & 1 & 2 & 1 & 10 & 3 & 2 & 1 & 2 \\
$a b c d, a b, a c$ & 8 & 1 & 2 & 1 & 8 & 4 & 2 & 0 & 3 \\
$a b d, a c, b c$ & 7 & 1 & 3 & 0 & 11 & 2 & 3 & 1 & 4 \\
$a b c, a b, a d$ & 7 & 2 & 1 & 1 & 11 & 2 & 3 & 1 & 5 \\
$a b c, a b d, a$ & 7 & 2 & 1 & 1 & 9 & 3 & 3 & 0 & 6 \\
$a b, a c, b d$ & 6 & 2 & 2 & 0 & 12 & 2 & 2 & 2 & 7 \\
$a b c, a d, b$ & 6 & 2 & 2 & 0 & 10 & 3 & 2 & 1 & 8 \\
$a b, a c, a d$ & 6 & 3 & 0 & 1 & 12 & 0 & 6 & 0 & 9 \\
$a b, a c, d$ & 5 & 3 & 1 & 0 & 11 & 2 & 3 & 1 & 10 \\
\hline
\end{tabular}

${ }^{a}$ replicate generator

Table A.11: Designs in $\mathcal{D}_{3}^{p}$, for $p=5,6$ and 7

\begin{tabular}{|c|c|c|c|c|c|c|c|c|c|}
\hline \multicolumn{10}{|c|}{$p=5$, designs have 96 runs } \\
\hline $\mathrm{rg}^{a}$ sets & $N$ & $n_{1}$ & $n_{2}$ & $n_{3}$ & $T$ & $t_{1}$ & $t_{2}$ & $t_{3}$ & ranking \\
\hline$a b c e, a b d, a c d$ & 10 & 1 & 3 & 1 & 16 & 5 & 4 & 1 & 1 \\
\hline$a b c, a b d, a c e$ & 9 & 2 & 2 & 1 & 18 & 4 & 4 & 2 & 2 \\
\hline$a b c d, a b e, a c$ & 9 & 2 & 2 & 1 & 16 & 5 & 4 & 1 & 3 \\
\hline$a b d, a c e, b c$ & 8 & 2 & 3 & 0 & 18 & 4 & 4 & 2 & 4 \\
\hline$a b c, a b d, a e$ & 8 & 3 & 1 & 1 & 18 & 3 & 6 & 1 & 5 \\
\hline$a b c, a d, b e$ & 7 & 3 & 2 & 0 & 18 & 4 & 4 & 2 & 6 \\
\hline \multicolumn{10}{|c|}{$p=6$, designs have 192 runs } \\
\hline $\mathrm{rg}^{a}$ sets & $N$ & $n_{1}$ & $n_{2}$ & $n_{3}$ & $T$ & $t_{1}$ & $t_{2}$ & $t_{3}$ & ranking \\
\hline$a b c e, a b d f, a c d$ & 11 & 2 & 3 & 1 & 25 & 7 & 6 & 2 & 1 \\
\hline$a b c d$, abe, $a c f$ & 10 & 3 & 2 & 1 & 26 & 6 & 7 & 2 & 2 \\
\hline$a b d, a c e, b c f$ & 9 & 3 & 3 & 0 & 27 & 6 & 6 & 3 & 3 \\
\hline \multicolumn{10}{|c|}{$p=7$, design has 384 runs } \\
\hline $\mathrm{rg}^{a}$ sets & $N$ & $n_{1}$ & $n_{2}$ & $n_{3}$ & $T$ & $t_{1}$ & $t_{2}$ & $t_{3}$ & ranking \\
\hline$a b c e, a b d f, a c d g$ & 12 & 3 & 3 & 1 & 27 & 6 & 6 & 3 & 1 \\
\hline
\end{tabular}

${ }^{a}$ replicate generator 
Table A.12: $p=8$, designs in $\mathcal{D}_{4}^{8}$

\begin{tabular}{|c|c|c|c|c|c|c|c|c|c|c|c|}
\hline replicate generator sets & $N$ & $n_{1}$ & $n_{2}$ & $n_{3}$ & $n_{4}$ & $T$ & $t_{1}$ & $t_{2}$ & $t_{3}$ & $t_{4}$ & ranking \\
\hline$a b c d f g$, abcefh, abdeg, acdeh & 22 & 0 & 3 & 4 & 1 & 54 & 10 & 11 & 6 & 1 & 1 \\
\hline$a b c d f g h, a b c e f, a b d e g$, acdeh & 22 & 0 & 3 & 4 & 1 & 52 & 10 & 12 & 6 & 0 & $=2$ \\
\hline$a b c d f g$, abce $f h$, abdegh, acde & 22 & 0 & 3 & 4 & 1 & 52 & 10 & 12 & 6 & 0 & $=2$ \\
\hline$a b c d e, a b c e f g, a b d f h, a c d g h$ & 21 & 0 & 4 & 3 & 1 & 57 & 8 & 12 & 7 & 1 & 4 \\
\hline$a b c d e f, a b c e g, a b d f h, a c d g h$ & 21 & 0 & 4 & 3 & 1 & 57 & 9 & 11 & 6 & 2 & 5 \\
\hline$a b c d e f, a b c e g h, a b d f g, a c d h$ & 21 & 0 & 4 & 3 & 1 & 55 & 9 & 12 & 6 & 1 & 6 \\
\hline$a b c d e f g, a b c e h, a b d f h, a c d g$ & 21 & 0 & 4 & 3 & 1 & 53 & 10 & 12 & 5 & 1 & 7 \\
\hline abcdf $g$, abcef, abdeg, acdeh & 21 & 1 & 2 & 4 & 1 & 57 & 8 & 12 & 7 & 1 & 8 \\
\hline$a b c d f h$, abcef, abdeg, acdeg & 21 & 1 & 2 & 4 & 1 & 57 & 9 & 11 & 6 & 2 & 9 \\
\hline$a b c d f g$, abcef $h$, abdeg, acde & 21 & 1 & 2 & 4 & 1 & 55 & 9 & 12 & 6 & 1 & 10 \\
\hline$a b c d f g h$, abce $f$, abdeg, acde & 21 & 1 & 2 & 4 & 1 & 53 & 10 & 12 & 5 & 1 & 11 \\
\hline abce $f$, abdeg, acdf $h, b c d g h$ & 20 & 0 & 4 & 4 & 0 & 60 & 8 & 10 & 8 & 2 & 12 \\
\hline$a b c e f g, a b d e h, a c d f h, b c d g$ & 20 & 0 & 4 & 4 & 0 & 58 & 8 & 11 & 8 & 1 & 13 \\
\hline$a b c d e, a b c f g, a b d f h$, acegh & 20 & 0 & 5 & 2 & 1 & 60 & 6 & 14 & 6 & 2 & 14 \\
\hline abcdef, abcdg, abegh, acfh & 20 & 0 & 5 & 2 & 1 & 58 & 7 & 14 & 5 & 2 & 15 \\
\hline$a b c d e f g h, a b c e, a b d f, a c d g^{a}$ & 20 & 1 & 3 & 3 & 1 & 48 & 12 & 12 & 4 & 0 & 33 \\
\hline$a b e, a c f, b d g, c d h$ & 12 & 4 & 4 & 0 & 0 & 58 & 8 & 11 & 8 & 1 & 365 \\
\hline
\end{tabular}

${ }^{a}$ Kerr design

Table A.13: $p=9$, designs in $\mathcal{D}_{4}^{9}$

\begin{tabular}{lccccccccccc}
\hline replicate generator sets & $N$ & $n_{1}$ & $n_{2}$ & $n_{3}$ & $n_{4}$ & $T$ & $t_{1}$ & $t_{2}$ & $t_{3}$ & $t_{4}$ & ranking \\
\hline abcdfg, abcefh, abdegi, acdehi & 24 & 0 & 4 & 4 & 1 & 72 & 12 & 14 & 8 & 2 & 1 \\
abcdfgh, abcefi, abdegi, acdeh & 24 & 0 & 4 & 4 & 1 & 70 & 12 & 15 & 8 & 1 & 2 \\
abcdef, abcegh, abdfgi, acdhi & 23 & 0 & 5 & 3 & 1 & 74 & 10 & 16 & 8 & 2 & 3 \\
abcdefg, abcehi, abdfh, acdgi & 23 & 0 & 5 & 3 & 1 & 72 & 11 & 16 & 7 & 2 & 4 \\
abcdfg, abcefh, abdegh, acdei & 23 & 1 & 3 & 4 & 1 & 74 & 10 & 15 & 10 & 1 & 5 \\
abcdfg, abcefh, abdegi, acdeh & 23 & 1 & 3 & 4 & 1 & 74 & 11 & 14 & 9 & 2 & 6 \\
abcdfgh, abcefi, abdeg, acdeh & 23 & 1 & 3 & 4 & 1 & 72 & 11 & 15 & 9 & 1 & 7 \\
abcdfgi, abcefh, abdeg, acdeh & 23 & 1 & 3 & 4 & 1 & 72 & 12 & 14 & 8 & 2 & 8 \\
abcdfgi, abcefh, abdegh, acde & 23 & 1 & 3 & 4 & 1 & 70 & 12 & 15 & 8 & 1 & 9 \\
abcdfghi, abcef, abdeg, acdeh & 23 & 1 & 3 & 4 & 1 & 68 & 13 & 15 & 7 & 1 & 10 \\
abcefg, abdehi, acdfh, bcdgi & 22 & 0 & 5 & 4 & 0 & 76 & 10 & 14 & 10 & 2 & 11 \\
abcdef, abcdgh, abegi, acf $h i$ & 22 & 0 & 6 & 2 & 1 & 76 & 8 & 19 & 6 & 3 & 12 \\
abcdei, abcefg, abdf $h$, acdgh & 22 & 1 & 4 & 3 & 1 & 76 & 9 & 15 & 11 & 1 & 13 \\
abcdef, abcegh, abdfg, acdhi & 22 & 1 & 4 & 3 & 1 & 76 & 10 & 14 & 10 & 2 & $=14$ \\
abcde, abcefg, abdf $i$, acdgh & 22 & 1 & 4 & 3 & 1 & 76 & 10 & 14 & 10 & 2 & $=14$ \\
abcf, adeg, bdh, cei & 14 & 4 & 5 & 0 & 0 & 76 & 10 & 14 & 10 & 2 & 290 \\
\hline
\end{tabular}


Table A.14: $p=10$, designs in $\mathcal{D}_{4}^{10}$

\begin{tabular}{lccccccccccc}
\hline replicate generator sets & $N$ & $n_{1}$ & $n_{2}$ & $n_{3}$ & $n_{4}$ & $T$ & $t_{1}$ & $t_{2}$ & $t_{3}$ & $t_{4}$ & ranking \\
\hline abcdfgh, abcefij, abdegi, acdehj & 26 & 0 & 5 & 4 & 1 & 90 & 14 & 19 & 10 & 2 & 1 \\
abcdefg, abcehi, abdfhj, acdgij & 25 & 0 & 6 & 3 & 1 & 93 & 12 & 21 & 9 & 3 & 2 \\
abcdfgh, abcefi, abdegi, acdehj & 25 & 1 & 4 & 4 & 1 & 93 & 13 & 18 & 12 & 2 & 3 \\
abcdfgj, abcefh, abdegi, acdehi & 25 & 1 & 4 & 4 & 1 & 93 & 14 & 17 & 11 & 3 & 4 \\
abcdfgh, abcefij, abdegi, acdeh & 25 & 1 & 4 & 4 & 1 & 91 & 14 & 18 & 11 & 2 & 5 \\
abcdfghj, abcefi, abdegi, acdeh & 25 & 1 & 4 & 4 & 1 & 89 & 15 & 18 & 10 & 2 & 6 \\
abcdefj, abcegh, abdfgi, acdhi & 24 & 1 & 5 & 3 & 1 & 94 & 12 & 19 & 12 & 2 & 7 \\
abcdefg, abcehi, abdfhj, acdgi & 24 & 1 & 5 & 3 & 1 & 94 & 13 & 18 & 11 & 3 & $=8$ \\
abcdef, abceghj, abdfgi, acdhi & 24 & 1 & 5 & 3 & 1 & 94 & 13 & 18 & 11 & 3 & $=8$ \\
abcdefg, abcehij, abdfh, acdgi & 24 & 1 & 5 & 3 & 1 & 92 & 14 & 18 & 10 & 3 & 10 \\
abcdefgj, abcehi, abdf $h$, acdgi & 24 & 1 & 5 & 3 & 1 & 90 & 14 & 19 & 10 & 2 & 11 \\
abcdfg, abcefh, abdegi, acdehj $j$ & 24 & 2 & 3 & 4 & 1 & 96 & 12 & 18 & 12 & 3 & 12 \\
abcdfgh, abcefi, abdegj, acdeh & 24 & 2 & 3 & 4 & 1 & 94 & 12 & 19 & 12 & 2 & $=13$ \\
abcdfgi, abcef $h$, abdegh, acdej $j$ & 24 & 2 & 3 & 4 & 1 & 94 & 12 & 19 & 12 & 2 & $=13$ \\
abcdfgi, abcef $h$, abdegj, acdeh & 24 & 2 & 3 & 4 & 1 & 94 & 13 & 18 & 11 & 3 & $=15$ \\
abcdfgi, abcefh, abdeg, acdehj & 24 & 2 & 3 & 4 & 1 & 94 & 13 & 18 & 11 & 3 & $=15$ \\
abcg, adeh, bdfi, cefj & 16 & 4 & 6 & 0 & 0 & 96 & 12 & 18 & 12 & 3 & 187 \\
\hline
\end{tabular}

Table A.15: $p=11$, designs in $\mathcal{D}_{4}^{11}$

\begin{tabular}{lccccccccccc}
\hline replicate generator sets & $N$ & $n_{1}$ & $n_{2}$ & $n_{3}$ & $n_{4}$ & $T$ & $t_{1}$ & $t_{2}$ & $t_{3}$ & $t_{4}$ & ranking \\
\hline abcdfgh, abcefij, abdegik, abdehjk & 28 & 0 & 6 & 4 & 1 & 112 & 16 & 24 & 12 & 3 & 1 \\
abcdfgh, abcefij, abdegik, acdehj & 27 & 1 & 5 & 4 & 1 & 114 & 16 & 22 & 14 & 3 & 2 \\
abcdfghk, abcefij, abdegi, acdehj & 27 & 1 & 5 & 4 & 1 & 112 & 17 & 22 & 13 & 3 & 3 \\
abcdefg, abcehik, abdfhj, acdgij & 26 & 1 & 6 & 3 & 1 & 116 & 15 & 23 & 13 & 4 & 4 \\
abcdefgk, abcehi, abdfhj, acdgij & 26 & 1 & 6 & 3 & 1 & 114 & 15 & 24 & 13 & 3 & 5 \\
abcdfgh, abcefij, abdegi, acdehk & 26 & 2 & 4 & 4 & 1 & 116 & 15 & 22 & 15 & 3 & 6 \\
abcdfgj, abcefhk, abdegi, acdehi & 26 & 2 & 4 & 4 & 1 & 116 & 16 & 21 & 14 & 4 & $=7$ \\
abcdfgj, abcefh, abdegi, acdehik & 26 & 2 & 4 & 4 & 1 & 116 & 16 & 21 & 14 & 4 & $=7$ \\
abcdfghj, abcefi, abdegi, acdehk & 26 & 2 & 4 & 4 & 1 & 114 & 16 & 22 & 14 & 3 & $=9$ \\
abcdfgh, abcefij, abdegik, acdeh & 26 & 2 & 4 & 4 & 1 & 114 & 16 & 22 & 14 & 3 & $=9$ \\
abcdfghj, abcefik, abdegi, acdeh & 26 & 2 & 4 & 4 & 1 & 112 & 17 & 22 & 13 & 3 & 11 \\
abcefgk, abdehi, acdfhj, bcdgij & 25 & 1 & 6 & 4 & 0 & 118 & 15 & 21 & 15 & 4 & 12 \\
abcdefj, abcegh, abdfgi, acdhik & 25 & 2 & 5 & 3 & 1 & 118 & 14 & 22 & 16 & 3 & 13 \\
abcdefg, abcehi, abdf $h j$, acdgik & 25 & 2 & 5 & 3 & 1 & 118 & 15 & 21 & 15 & 4 & $=14$ \\
abcdef, abceghj, abdfgi, acdhik & 25 & 2 & 5 & 3 & 1 & 118 & 15 & 21 & 15 & 4 & $=14$ \\
abdh, abefi, acegi, dfgk & 19 & 4 & 6 & 1 & 0 & 118 & 15 & 21 & 15 & 4 & 97 \\
\hline
\end{tabular}


Table A.16: $p=12$, designs in $\mathcal{D}_{4}^{12}$

\begin{tabular}{lccccccccccc}
\hline replicate generator sets & $N$ & $n_{1}$ & $n_{2}$ & $n_{3}$ & $n_{4}$ & $T$ & $t_{1}$ & $t_{2}$ & $t_{3}$ & $t_{4}$ & ranking \\
\hline abcdfghl, abcefij, abdegik, abdehjk & 29 & 1 & 6 & 4 & 1 & 137 & 19 & 27 & 16 & 4 & 1 \\
abcdfgh, abcefij, abdegik, acdehjl & 28 & 2 & 5 & 4 & 1 & 140 & 18 & 26 & 18 & 4 & 2 \\
abcdfghk, abcefij, abdegil, acdehj & 28 & 2 & 5 & 4 & 1 & 138 & 19 & 26 & 17 & 4 & 3 \\
abcdfghk, abcefijl, abdegi, acdehj & 28 & 2 & 5 & 4 & 1 & 136 & 20 & 26 & 16 & 4 & 4 \\
abcdefg, abcehik, abdfhjl, acdgij & 27 & 2 & 6 & 3 & 1 & 141 & 18 & 26 & 17 & 5 & 5 \\
abcdefgk, abcehil, abdfhj, acdgij & 27 & 2 & 6 & 3 & 1 & 139 & 18 & 27 & 17 & 4 & 6 \\
abcdfgh, abcefij, abdegik, acdehl & 27 & 3 & 4 & 4 & 1 & 141 & 17 & 27 & 18 & 4 & 7 \\
abcdfgj, abcefhk, abdegil, acdehi & 27 & 3 & 4 & 4 & 1 & 141 & 18 & 26 & 17 & 5 & 8 \\
abcdfghj, abcefik, abdegi, acdehl & 27 & 3 & 4 & 4 & 1 & 139 & 18 & 27 & 17 & 4 & 9 \\
abcdfghj, abcefik, abdegil, acdeh & 27 & 3 & 4 & 4 & 1 & 137 & 19 & 27 & 16 & 4 & 10 \\
abcefgk, abdehil, acdfhj, bcdgij & 26 & 2 & 6 & 4 & 0 & 142 & 18 & 25 & 18 & 5 & 11 \\
abcdefj, abceghk, abdfgi, acdhil & 26 & 3 & 5 & 3 & 1 & 142 & 17 & 26 & 19 & 4 & 12 \\
abcdefg, abcehij, abdfhk, acdgil & 26 & 3 & 5 & 3 & 1 & 142 & 18 & 25 & 18 & 5 & $=13$ \\
abcdef, abceghj, abdfgik, acdhil & 26 & 3 & 5 & 3 & 1 & 142 & 18 & 25 & 18 & 5 & $=13$ \\
abcdefgj, abcehi, abdfhk, acdgil & 26 & 3 & 5 & 3 & 1 & 140 & 18 & 26 & 18 & 4 & $=15$ \\
abcdefj, abceghk, abdfgil, acdhi & 26 & 3 & 5 & 3 & 1 & 140 & 18 & 26 & 18 & 4 & $=15$ \\
abcdei, abcfgj, adf hk, beghl & 22 & 4 & 6 & 2 & 0 & 142 & 18 & 25 & 18 & 5 & 39 \\
\hline
\end{tabular}


Table A.17: Designs in $\mathcal{D}_{4}^{p}$, for $p=13,14$ and 15

\begin{tabular}{|c|c|c|c|c|c|c|c|c|c|c|c|}
\hline replicate generator sets & $N$ & $n_{1}$ & $n_{2}$ & $n_{3}$ & $n_{4}$ & $T$ & $t_{1}$ & $t_{2}$ & $t_{3}$ & $t_{4}$ & ranking \\
\hline abcdfghi, abcefijm, abdegik, acdehjk & 30 & 2 & 6 & 4 & 1 & 164 & 22 & 31 & 20 & 5 & 1 \\
\hline abcdfghk, abcefij, abdegil, acdehjm & 29 & 3 & 5 & 4 & 1 & 166 & 21 & 31 & 21 & 5 & 2 \\
\hline abcdfghk, abcefijl, abdegim, acdehj & 29 & 3 & 5 & 4 & 1 & 164 & 22 & 31 & 20 & 5 & 3 \\
\hline abcdefg, abcehik, abdfhjl, acdgijm & 28 & 3 & 6 & 3 & 1 & 168 & 21 & 30 & 21 & 6 & 4 \\
\hline abcdefgk, abcehil, abdfhjm, acdgij & 28 & 3 & 6 & 3 & 1 & 166 & 21 & 31 & 21 & 5 & 5 \\
\hline abcdfgj, abcefhk, abdegil, acdehim & 28 & 4 & 4 & 4 & 1 & 168 & 20 & 32 & 20 & 6 & 6 \\
\hline abcdfghj, abcefik, abdegil, acdehm & 28 & 4 & 4 & 4 & 1 & 166 & 20 & 33 & 20 & 5 & 7 \\
\hline abcefgk, abdehil, acdfhjm, bcdgij & 27 & 3 & 6 & 4 & 0 & 168 & 21 & 30 & 21 & 6 & 8 \\
\hline abcdefj, abceghk, abdfgil, acdhim & 27 & 4 & 5 & 3 & 1 & 168 & 20 & 31 & 22 & 5 & 9 \\
\hline abcdefgj, abcehik, abdfhl, acdgim & 27 & 4 & 5 & 3 & 1 & 166 & 21 & 31 & 21 & 5 & 10 \\
\hline abcefgj, abdehik, acdfhl, bcdgim & 26 & 4 & 5 & 4 & 0 & 168 & 20 & 32 & 20 & 6 & 11 \\
\hline abcdefj, abcdghk, abegil, acfhim & 26 & 4 & 6 & 2 & 1 & 168 & 20 & 31 & 22 & 5 & 12 \\
\hline abcdef $j$, abdghk, acegil, bcfhim & 25 & 4 & 6 & 3 & 0 & 168 & 21 & 30 & 21 & 6 & 13 \\
\hline \multicolumn{12}{|c|}{$p=14$} \\
\hline replicate generator sets & $N$ & $n_{1}$ & $n_{2}$ & $n_{3}$ & $n_{4}$ & $T$ & $t_{1}$ & $t_{2}$ & $t_{3}$ & $t_{4}$ & ranking \\
\hline abcdfghl, abcefijm, abdegikn, acdehjk & 31 & 3 & 6 & 4 & 1 & 193 & 25 & 36 & 24 & 6 & 1 \\
\hline abcdfghk, abcefijl, abdegim, acdehjn & 30 & 4 & 5 & 4 & 1 & 194 & 24 & 37 & 24 & 6 & 2 \\
\hline abcdefgk, abcehil, abdfhjm, acdgijn & 29 & 4 & 6 & 3 & 1 & 195 & 24 & 36 & 25 & 6 & 3 \\
\hline abcefgk, abdehil, acdfhjm, bcdgijn & 28 & 4 & 6 & 4 & 0 & 196 & 24 & 36 & 24 & 7 & 4 \\
\hline \multicolumn{12}{|c|}{$p=15$} \\
\hline replicate generator sets & $N$ & $n_{1}$ & $n_{2}$ & $n_{3}$ & $n_{4}$ & $T$ & $t_{1}$ & $t_{2}$ & $t_{3}$ & $t_{4}$ & ranking \\
\hline abcdfghl, abcefijm, abdegikn, acdehjko & 32 & 4 & 6 & 4 & 1 & 224 & 28 & 42 & 28 & 7 & 1 \\
\hline
\end{tabular}

